\title{
Searches for low-mass dimuon resonances
}

\section{IHCW}

\section{The LHCb collaboration}

\section{E-mail: mwill@mit.edu}

ABSTRACT: Searches are performed for a low-mass dimuon resonance, $X$, produced in proton-proton collisions at a center-of-mass energy of $13 \mathrm{TeV}$, using a data sample corresponding to an integrated luminosity of $5.1 \mathrm{fb}^{-1}$ and collected with the LHCb detector. The $X$ bosons can either decay promptly or displaced from the proton-proton collision, where in both cases the requirements placed on the event and the assumptions made about the production mechanisms are kept as minimal as possible. The searches for promptly decaying $X$ bosons explore the mass range from near the dimuon threshold up to $60 \mathrm{GeV}$, with nonnegligible $X$ widths considered above $20 \mathrm{GeV}$. The searches for displaced $X \rightarrow \mu^{+} \mu^{-}$ decays consider masses up to $3 \mathrm{GeV}$. None of the searches finds evidence for a signal and $90 \%$ confidence-level exclusion limits are placed on the $X \rightarrow \mu^{+} \mu^{-}$cross sections, each with minimal model dependence. In addition, these results are used to place world-leading constraints on $\mathrm{GeV}$-scale bosons in the two-Higgs-doublet and hidden-valley scenarios.

Keywords: Beyond Standard Model, Exotics, Hadron-Hadron scattering (experiments)

ARXIV EPRINT: 2007.03923 


\section{Contents}

1 Introduction 1

2 Detector and simulation $\quad 3$

$\begin{array}{lll}3 & \text { Selection } & 4\end{array}$

4 Signal searches $\quad 6$

$5 \quad$ Efficiency and luminosity $\quad 10$

6 Cross-section results 12

$\begin{array}{lll}7 & \text { Summary } & 16\end{array}$

$\begin{array}{lr}\text { The LHCb collaboration } & 20\end{array}$

\section{Introduction}

Substantial effort has been dedicated [1] to searching for a massive dark photon, $A^{\prime}$, which obtains a small coupling to the electromagnetic current due to kinetic mixing between the Standard Model (SM) hypercharge and $A^{\prime}$ field strength tensors [2-9]. However, this minimal $A^{\prime}$ model is not the only viable dark-sector scenario. The strongest connection to the dark sector may not arise via kinetic mixing, and the dark sector itself could be populated by additional particles that have phenomenological implications. Searches for dark photons can provide serendipitous discovery potential for other types of particles, generically labeled here as $X$ bosons, especially vector particles that share the same production mechanisms as the minimal dark photon [10], yet many well-motivated types of $X$ bosons would have avoided detection in all previous experimental searches [11, 12]. For example, hidden-valley (HV) scenarios that exhibit confinement produce a high multiplicity of light hidden hadrons from showering processes [13]. These hidden hadrons would typically decay displaced from the proton-proton collision, thus failing the criteria employed in refs. [14, 15] to suppress backgrounds due to heavy-flavor quarks $[16,17]$. Furthermore, the sensitivity to various model scenarios can be improved by exploiting additional signatures, e.g., the presence of a $b$-quark jet produced in association with the $X$ boson [18]. Therefore, it is desirable to perform searches that are less model dependent, including some that explore additional signatures in the event.

This article presents searches for low-mass dimuon resonances produced in protonproton collisions at a center-of-mass energy of $13 \mathrm{TeV}$, using a data sample corresponding to an integrated luminosity of $5.1 \mathrm{fb}^{-1}$ and collected with the LHCb detector in 2016-2018. The $X$ bosons can either decay promptly or displaced from the proton-proton collision. 
In both cases, the requirements placed on the event and the assumptions made about the production mechanisms are kept as minimal as possible. Two variations of the search for prompt $X \rightarrow \mu^{+} \mu^{-}$decays are performed: an inclusive version, and an $X+b$ search, where the $X$ boson is required to be produced in association with a beauty quark. Two variations are also considered of the search for displaced $X \rightarrow \mu^{+} \mu^{-}$decays: an inclusive version, and one where the $X$ boson is required to be produced promptly in the proton-proton collision. The searches for prompt $X \rightarrow \mu^{+} \mu^{-}$decays explore the mass range from near the dimuon threshold up to $60 \mathrm{GeV}$ (natural units with $c=1$ are implied throughout this article), with nonnegligible widths, $\Gamma(X)$, considered above $20 \mathrm{GeV}$. The searches for displaced $X \rightarrow \mu^{+} \mu^{-}$decays consider masses up to $3 \mathrm{GeV}$. This analysis uses the same data sample as the LHCb minimal dark-photon search [15]; however, the searches presented here are roughly half as sensitive to the minimal $A^{\prime}$ model, since the fiducial regions and selection criteria are not optimized for that scenario. These searches are much more sensitive for many other $X$ boson scenarios, including HV models.

The fiducial regions used for each search, defined in table 1, ensure that the detector response is sufficiently model independent in the kinematic regions where results are reported. The requirements placed on the momenta, $p$, and transverse momenta, $p_{\mathrm{T}}$, of the muons make them sufficiently energetic to be selected by the trigger, but not so energetic that their charges cannot be determined. Only events with at least one reconstructed proton-proton primary vertex (PV) are used in the analysis, which requires that at least five charged prompt particles, including the muons if the $X$ decays promptly, are produced in the same collision as the $X$ boson. A maximum number of charged particles is allowed to be produced in the collision, since the detector response depends on the charged-particle multiplicity. In practice, this maximum value is sufficiently large to have no impact on any of the scenarios considered here. The dimuon opening angle is required to be $\alpha\left(\mu^{+} \mu^{-}\right)>1(3) \mathrm{mrad}$ in the searches for prompt (displaced) $X \rightarrow \mu^{+} \mu^{-}$decays to ensure that the reconstruction efficiency factorizes into the product of the two individual muon efficiencies, which subsequently leads to an upper limit on $p_{\mathrm{T}}(X)$ to remove regions where the $\alpha\left(\mu^{+} \mu^{-}\right)$requirement is rarely satisfied. The $X+b$ analysis is performed using jets clustered with the anti- $k_{\mathrm{T}}$ algorithm [19] using a distance parameter $R=0.5$. The jets are required to have $20<p_{\mathrm{T}}$ (jet) $<100 \mathrm{GeV}$ and a pseudorapidity in the range $2.2<\eta($ jet $)<4.2$ so that the $b$-tagging efficiency is nearly uniform within the fiducial region. Finally, the displaced $X \rightarrow \mu^{+} \mu^{-}$secondary vertex (SV) is required to be transversely displaced from the PV in the range $12<\rho_{\mathrm{T}}<30 \mathrm{~mm}$, which results in minimal dependence on the SV location distribution. For example, this requirement leads to the efficiency being nearly independent of the $X$ lifetime, $\tau(X)$; however, the probability that the $X$ boson decays in this region is strongly dependent on $\tau(X)$.

This article is structured as follows. The LHCb detector, trigger, and simulation are described in section 2, while the offline selections used in each of the searches are discussed in section 3. Section 4 presents the searches for both prompt and displaced $X \rightarrow \mu^{+} \mu^{-}$ decays. Section 5 discusses the efficiencies and luminosity. The model-independent crosssection results, along with their interpretations within the context of specific models, are described in section 6 . Section 7 provides a summary and discussion of all results. 


\begin{tabular}{|c|c|}
\hline All searches & $\begin{array}{c}p_{\mathrm{T}}(\mu)>0.5 \mathrm{GeV} \\
10<p(\mu)<1000 \mathrm{GeV} \\
2<\eta(\mu)<4.5 \\
\sqrt{p_{\mathrm{T}}\left(\mu^{+}\right) p_{\mathrm{T}}\left(\mu^{-}\right)}>1 \mathrm{GeV} \\
5 \leq n_{\text {charged }}(2<\eta<4.5, p>5 \mathrm{GeV})<100(\text { from same } \mathrm{PV} \text { as } X)\end{array}$ \\
\hline $\begin{array}{c}\text { Prompt } \\
X \rightarrow \mu^{+} \mu^{-} \text {decays }\end{array}$ & $\begin{array}{c}1<p_{\mathrm{T}}(X)<50 \mathrm{GeV} \\
X \text { decay time }<0.1 \mathrm{ps} \\
\alpha\left(\mu^{+} \mu^{-}\right)>1 \mathrm{mrad} \\
20<p_{\mathrm{T}}(b \text {-jet })<100 \mathrm{GeV}, 2.2<\eta(b \text {-jet })<4.2(X+b \text { only })\end{array}$ \\
\hline $\begin{array}{c}\text { Displaced } \\
X \rightarrow \mu^{+} \mu^{-} \text {decays }\end{array}$ & $\begin{array}{c}2<p_{\mathrm{T}}(X)<10 \mathrm{GeV} \\
2<\eta(X)<4.5 \\
\alpha\left(\mu^{+} \mu^{-}\right)>3 \mathrm{mrad} \\
12<\rho_{\mathrm{T}}(X)<30 \mathrm{~mm} \\
X \text { produced in } p p \text { collision (promptly produced } X \text { only) }\end{array}$ \\
\hline
\end{tabular}

Table 1. Fiducial regions of the searches for prompt and displaced $X \rightarrow \mu^{+} \mu^{-}$decays.

\section{Detector and simulation}

The LHCb detector $[20,21]$ is a single-arm forward spectrometer covering the pseudorapidity range $2<\eta<5$, designed for the study of particles containing $b$ or $c$ quarks. The detector includes a high-precision tracking system consisting of a silicon-strip vertex detector surrounding the proton-proton interaction region (VELO), a large-area silicon-strip detector located upstream of a dipole magnet with a bending power of about $4 \mathrm{Tm}$, and three stations of silicon-strip detectors and straw drift tubes placed downstream of the magnet. The tracking system provides a measurement of the momentum of charged particles with a relative uncertainty that varies from $0.5 \%$ at low momentum to $1.0 \%$ at $200 \mathrm{GeV}$. The minimum distance of a track to a PV, the impact parameter, is measured with a resolution of $\left(15+29 / p_{\mathrm{T}}\right) \mu \mathrm{m}$, where $p_{\mathrm{T}}$ is in $\mathrm{GeV}$. Different types of charged hadrons are distinguished using information from two ring-imaging Cherenkov detectors. Photons, electrons and hadrons are identified by a calorimeter system consisting of scintillating-pad and preshower detectors, an electromagnetic and a hadronic calorimeter. Muons are identified by a system composed of alternating layers of iron and multiwire proportional chambers.

The online event selection is performed by a trigger, which consists of a hardware stage followed by a two-level software stage. In between the two software stages, an alignment and calibration of the detector is performed in near real-time and their results are used in the trigger [22]. The same alignment and calibration information is propagated to the offline reconstruction, ensuring consistent and high-quality particle identification information between the trigger and offline software. The identical performance of the online and offline reconstruction offers the opportunity to perform physics analyses directly using candidates reconstructed in the trigger [23, 24], which the searches for prompt $X \rightarrow \mu^{+} \mu^{-}$ decays exploit. 
At the hardware trigger stage, events are required to have a dimuon pair with $p_{\mathrm{T}}\left(\mu^{+}\right) p_{\mathrm{T}}\left(\mu^{-}\right) \gtrsim(1.5 \mathrm{GeV})^{2}$ and at most 900 hits in the scintillating-pad detector, which prevents high-occupancy events from dominating the processing time in the software trigger stages. The latter requirement is the main motivation for defining the maximum charged-particle multiplicity in table 1 . In the software stage, where the $p_{\mathrm{T}}$ resolution is substantially improved compared to the hardware stage, $X \rightarrow \mu^{+} \mu^{-}$candidates are built from two oppositely charged tracks that form a good-quality vertex and satisfy stringent muon-identification criteria. All searches require $p_{\mathrm{T}}(X)>1 \mathrm{GeV}$ and $2<\eta(\mu)<4.5$. The searches for prompt $X \rightarrow \mu^{+} \mu^{-}$decays use muons that are consistent with originating from the PV, with $p_{\mathrm{T}}(\mu)>1.0 \mathrm{GeV}$ and momentum $p(\mu)>20 \mathrm{GeV}$ in the 2016 data sample, and $p_{\mathrm{T}}(\mu)>0.5 \mathrm{GeV}, p(\mu)>10 \mathrm{GeV}$, and $p_{\mathrm{T}}\left(\mu^{+}\right) p_{\mathrm{T}}\left(\mu^{-}\right)>(1.0 \mathrm{GeV})^{2}$ in 2017-2018. The searches for displaced $X \rightarrow \mu^{+} \mu^{-}$decays use muons with $p_{\mathrm{T}}(\mu)>0.5 \mathrm{GeV}$ and $p(\mu)>10 \mathrm{GeV}$ that are inconsistent with originating from any $\mathrm{PV}$, and require $2<\eta(X)<4.5$. In addition, the search for a long-lived promptly produced $X$ boson requires a decay topology consistent with a dimuon resonance originating from a $\mathrm{PV}$.

Simulation is required to model the effects of the detector acceptance and its response to $X \rightarrow \mu^{+} \mu^{-}$decays. In the simulation, $p p$ collisions are generated using PythiA [25, 26] with a specific LHCb configuration [27]. Decays of unstable particles are described by EvtGen [28], in which final-state radiation is generated using Рнотоs [29]. The interaction of the generated particles with the detector, and its response, are implemented using the Geant4 toolkit [30,31] as described in ref. [32]. Simulation is also used to place constraints on specific models. Prompt limits for light-pseudoscalar models are set with next-to-next-to-leading order cross-sections from HigLu [33, 34] using the NNPDF3.0 PDF set [35], branching fractions from HDECAY [36, 37], and fiducial acceptances from Pythia [38]. Displaced limits for HV models are set with Pythia [38] using a running $\alpha_{\mathrm{HV}}$ scheme [39], and couplings from DARKCAST [10].

\section{Selection}

The selection criteria are largely applied online in the trigger and most are the same as those used in the LHCb minimal dark-photon search [15]. The prompt dimuon sample, i.e. the sample used in the searches for prompt $X \rightarrow \mu^{+} \mu^{-}$decays, selected by the trigger described in section 2 predominantly consists of genuine prompt dimuon pairs. The only selection criteria applied offline in the inclusive search for prompt $X \rightarrow \mu^{+} \mu^{-}$decays, $p_{\mathrm{T}}(X)<50 \mathrm{GeV}$ and $\alpha\left(\mu^{+} \mu^{-}\right)>1 \mathrm{mrad}$, are included in the definition of the fiducial region. In addition to these, the search for a promptly decaying $X$ boson produced in association with a beauty quark requires at least one $b$-tagged jet with $p_{\mathrm{T}}$ (jet) $>20 \mathrm{GeV}$ and $2.2<\eta$ (jet) $<4.2$. The jets are formed by clustering charged and neutral particle-flow candidates [40] using the anti- $k_{\mathrm{T}}$ clustering algorithm as implemented in FASTJET [41]. The $b$-tagging requires an SV in the jet that satisfies the criteria given in ref. [42]. Figure 1 shows the $m\left(\mu^{+} \mu^{-}\right)$distributions of both prompt dimuon data samples in bins of width $\sigma\left[m\left(\mu^{+} \mu^{-}\right)\right] / 2$, where $\sigma\left[m\left(\mu^{+} \mu^{-}\right)\right]$denotes the dimuon invariant-mass resolution which varies from $0.6 \mathrm{MeV}$ near threshold to $0.6 \mathrm{GeV}$ at $m\left(\mu^{+} \mu^{-}\right)=60 \mathrm{GeV}$. 


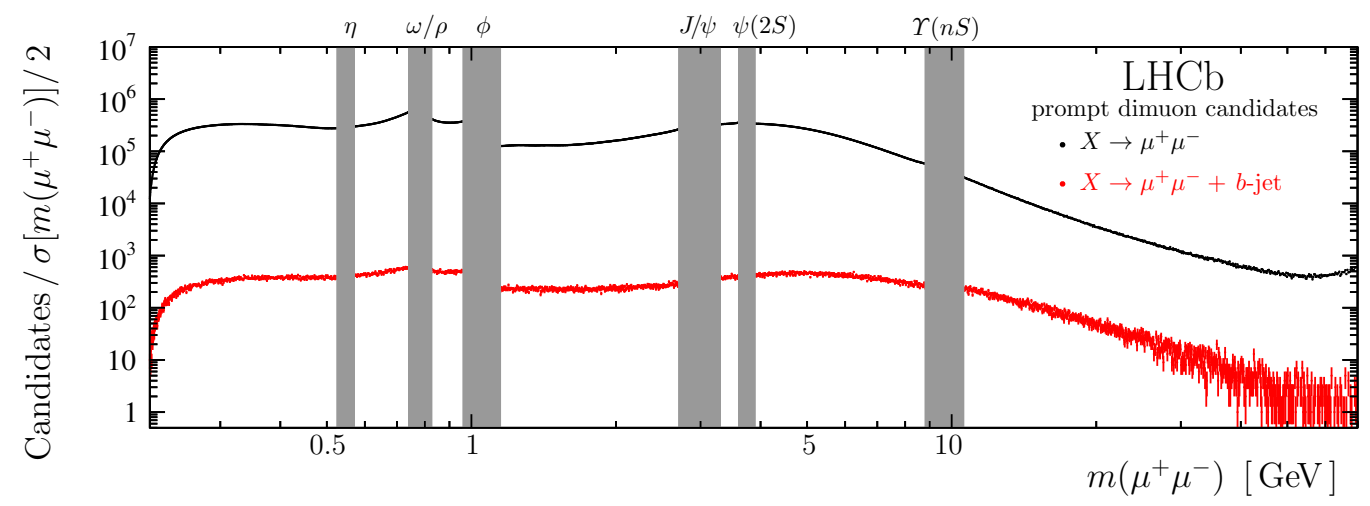

Figure 1. Prompt dimuon mass spectra showing the (black) inclusive and (red) $X+b$ candidates with all fiducial and selection requirements applied. The grey boxes show the regions vetoed due to large contributions from QCD resonances.

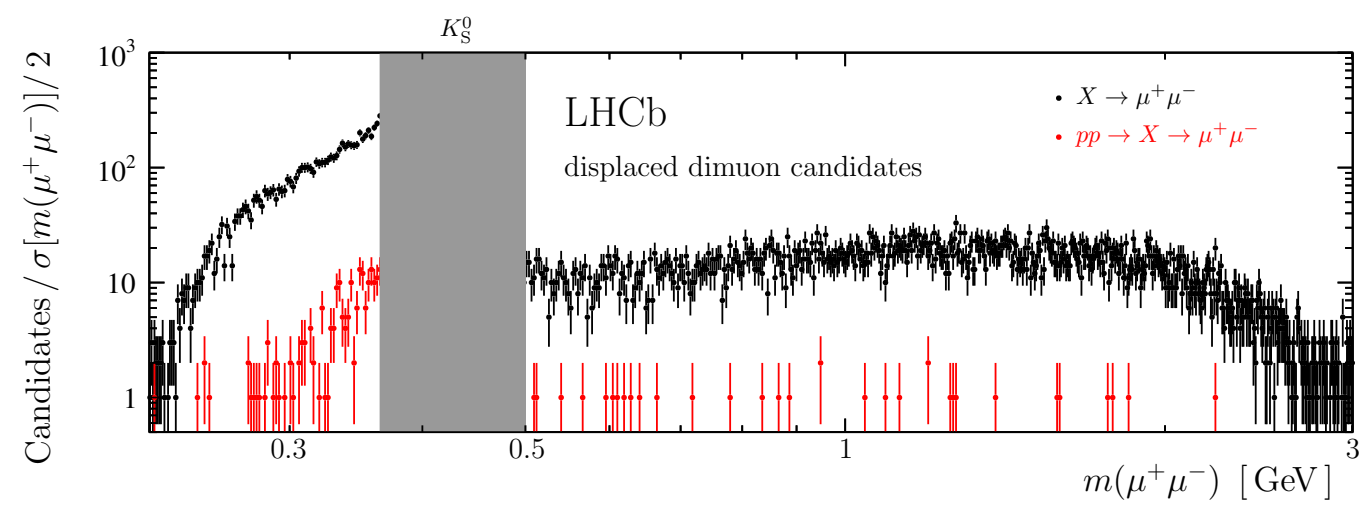

Figure 2. Displaced dimuon mass spectra showing the (black) inclusive and (red) promptly produced candidates with all fiducial and selection requirements applied. The grey box shows the region vetoed due to the large doubly misidentified $K_{\mathrm{S}}^{0}$ background, whose low-mass tail extends into the search region. A dedicated study of this region is presented in a search for $K_{\mathrm{S}}^{0} \rightarrow \mu^{+} \mu^{-}$decays [44].

In the searches for displaced $X \rightarrow \mu^{+} \mu^{-}$decays, contamination from prompt particles is negligible due to a stringent trigger criterion that requires muons to be inconsistent with originating from any PV. Furthermore, the fiducial region requires a transverse displacement from the $\mathrm{PV}$ of $12<\rho_{\mathrm{T}}<30 \mathrm{~mm}$, which is applied offline in both searches for displaced $X \rightarrow \mu^{+} \mu^{-}$decays and highly suppresses the background from $b$-hadron decay chains that produce two muons. Therefore, the dominant background contributions are due to material interactions in the VELO, e.g. photons that convert into $\mu^{+} \mu^{-}$pairs, and from $K_{\mathrm{S}}^{0} \rightarrow \pi^{+} \pi^{-}$decays, where both pions are misidentified as muons. A $p$-value is assigned to the material-interaction hypothesis for each displaced $X \rightarrow \mu^{+} \mu^{-}$candidate using properties of the SV and muon tracks, along with a high-precision three-dimensional material map produced from a data sample of secondary hadronic interactions [43]. The same mass-dependent requirement used in ref. [15] is applied to the $p$-values in this analysis, which highly suppresses the material-interaction background. Figure 2 shows the $m\left(\mu^{+} \mu^{-}\right)$ distributions of both displaced-dimuon data samples. 


\section{Signal searches}

The signal-search strategies and methods employed are similar to those used in ref. [15]. The dimuon mass spectra are scanned in around 6000 steps of about $\sigma\left[m\left(\mu^{+} \mu^{-}\right)\right] / 2$ searching for $X \rightarrow \mu^{+} \mu^{-}$contributions. For $m(X)<20 \mathrm{GeV}$, the data are binned in $p_{\mathrm{T}}(X)$ and each $p_{\mathrm{T}}$ bin is searched independently for each $m(X)$ hypothesis; whereas at higher masses, $p_{\mathrm{T}}$ bins are not necessary since both the resolution and efficiency are nearly independent of $p_{\mathrm{T}}(X)$. All searches use the profile likelihood method to determine the local $p$-values and the confidence intervals on the signal yields. The trial factors are obtained using pseudoexperiments in each search. The confidence intervals are defined using the bounded likelihood approach [45], which involves taking $\Delta \log \mathcal{L}$ relative to zero signal, rather than the best-fit value, if the best-fit signal value is negative. This approach enforces that only physical (nonnegative) upper limits are placed on the signal yields, and prevents defining exclusion regions that are much better than the experimental sensitivity in cases where a large deficit in the background yield is observed. The signal $m\left(\mu^{+} \mu^{-}\right)$distributions are well modeled by a Gaussian function, whose resolution is determined with $10 \%$ precision using a combination of simulated $X \rightarrow \mu^{+} \mu^{-}$decays and the observed $p_{\mathrm{T}^{-}}$-dependent widths of the large known resonance peaks present in the data. The mass-resolution uncertainty is included in the profile likelihood. The potential bias due to neglecting non-Gaussian components of the signal shape is much smaller than the uncertainty that arises from the limited knowledge of the mass resolution.

The fit strategy used in the searches for prompt $X \rightarrow \mu^{+} \mu^{-}$decays below $20 \mathrm{GeV}$, which is the same as in refs. [14, 15], was first introduced in ref. [46]. At each $m(X)$ hypothesis, a binned extended maximum-likelihood fit is performed in a $\pm 12.5 \sigma\left[m\left(\mu^{+} \mu^{-}\right)\right]$window around the $m(X)$ value. Near the dimuon threshold, the energy released in the decay, $Q=\sqrt{m\left(\mu^{+} \mu^{-}\right)^{2}-4 m(\mu)^{2}}$, is used instead of the mass because it is easier to model. The background model for each fit window takes as input a large set of potential components, then the data-driven model-selection process of ref. [46] is performed, whose uncertainty is included in the profile likelihood following ref. [47]. Specifically, the method labeled aic-o in ref. [46] is used, where the log-likelihood of each background model is penalized for its complexity (number of parameters). The confidence intervals are obtained from the profile likelihoods, including the penalty terms, where the model index is treated as a discrete nuisance parameter, as originally proposed in ref. [47]. In the $X+b$ search there are not many candidates near the dimuon threshold. Therefore, just in this region, the counting-experiment-based method of ref. [48] is used, which is also used in the searches for displaced $X \rightarrow \mu^{+} \mu^{-}$decays and described in detail below.

In this analysis, the set of possible background components is the same as in ref. [15] and includes all Legendre modes up to tenth order at every $m(X)$. Additionally, dedicated background components are included for sizable narrow SM resonance contributions. The use of 11 Legendre modes adequately describes every doubly misidentified peaking background that contributes at a significant level; therefore, these do not require dedicated background components. In mass regions where such complexity is not required, the data-driven model-selection procedure reduces the complexity, which increases the sensi- 
tivity to a potential signal contribution. Therefore, the impact of the background-model uncertainty on the size of the confidence intervals is mass dependent, though on average it is about $30 \%$. As in ref. [46], all fit regions are transformed onto the interval $[-1,1]$, where the $m(X)$ value is mapped to zero. After such a transformation, the signal model is (approximately) an even function; therefore, odd Legendre modes are orthogonal to the signal component, which means that the presence of odd modes has minimal impact on the variance of the observed signal yield. In the fits, all odd Legendre modes up to ninth order are included in every background model, while even modes must be selected for inclusion in each fit by the data-driven method of ref. [46].

Regions in the mass spectrum with large SM resonance contributions are vetoed in the searches for prompt $X \rightarrow \mu^{+} \mu^{-}$decays. Furthermore, the region near the $\eta^{\prime}$ meson is treated uniquely. Since it is not possible to distinguish between $X \rightarrow \mu^{+} \mu^{-}$and possible $\eta^{\prime} \rightarrow \mu^{+} \mu^{-}$contributions at $m\left(\eta^{\prime}\right)$, the $p$-values near this mass are ignored. The small observed excess at $m\left(\eta^{\prime}\right)$ is simply absorbed into the signal yield when setting the limits, which is conservative in that the $\eta^{\prime} \rightarrow \mu^{+} \mu^{-}$contribution weakens the constraints on $X \rightarrow \mu^{+} \mu^{-}$decays.

Figure 3 shows the signed local significances for all $m(X)$ below $20 \mathrm{GeV}$ for both searches for prompt $X \rightarrow \mu^{+} \mu^{-}$decays. The largest local excess in the inclusive search in this mass region is $3.7 \sigma$ at $349 \mathrm{MeV}$ in the $3<p_{\mathrm{T}}(X)<5 \mathrm{GeV}$ bin; however, its neighboring $p_{\mathrm{T}}$ bin at this mass has a small deficit and the global significance is only $\approx 1 \sigma$. Similarly, the largest local excess in the $X+b$ search below $20 \mathrm{GeV}$ is $3.1 \sigma$ at $2424 \mathrm{MeV}$ in the $10<p_{\mathrm{T}}(X)<20 \mathrm{GeV}$ bin, though again, the neighboring $p_{\mathrm{T}}$ bins both have deficits at the same mass, and the global significance is below $1 \sigma$. Therefore, no significant excess is found in either prompt spectrum for $m(X)<20 \mathrm{GeV}$.

In the $20<m(X)<60 \mathrm{GeV}$ region, the background is nearly monotonic, which permits the use of a simplified fit strategy. The entire $12<m\left(\mu^{+} \mu^{-}\right)<80 \mathrm{GeV}$ region is fitted when considering all $m(X)$ values above $20 \mathrm{GeV}$. The background model is comprised of three falling power-law terms and an eighth-order polynomial that collectively describe the DrellYan, heavy-flavor, and misidentified-background contributions, along with a rising powerlaw term to describe the low-mass tail of the $Z$ boson, where all parameters are free to vary. This background model is validated by studying simulated Drell-Yan dimuon production, same-sign dimuon data which predominantly consists of heavy-flavor and misidentification backgrounds, and candidates in the data sample itself above the search region. Unlike at lower masses, nonnegligible widths are considered. At each $m(X)$, a scan is performed covering the range $0 \leq \Gamma(X) \leq 3 \mathrm{GeV}$. The signals are modelled by a Gaussian resolution function convolved with the modulus of a Breit-Wigner function.

Figure 4 shows the signed local significances for the $m(X)>20 \mathrm{GeV}$ region for both searches for prompt $X \rightarrow \mu^{+} \mu^{-}$decays. The largest local excess in the inclusive search in this mass region is $3.2 \sigma$ at $m(X)=36 \mathrm{GeV}$ for $\Gamma(X)=1.5 \mathrm{GeV}$, which corresponds to a global $p$-value of about $11 \%$ (considering only the $m(X)>20 \mathrm{GeV}$ mass region). In the $X+b$ search, no local significance exceeds $\approx 2 \sigma$ in this mass region. Therefore, no significant excess is found in either prompt spectrum for $m(X)>20 \mathrm{GeV}$. 

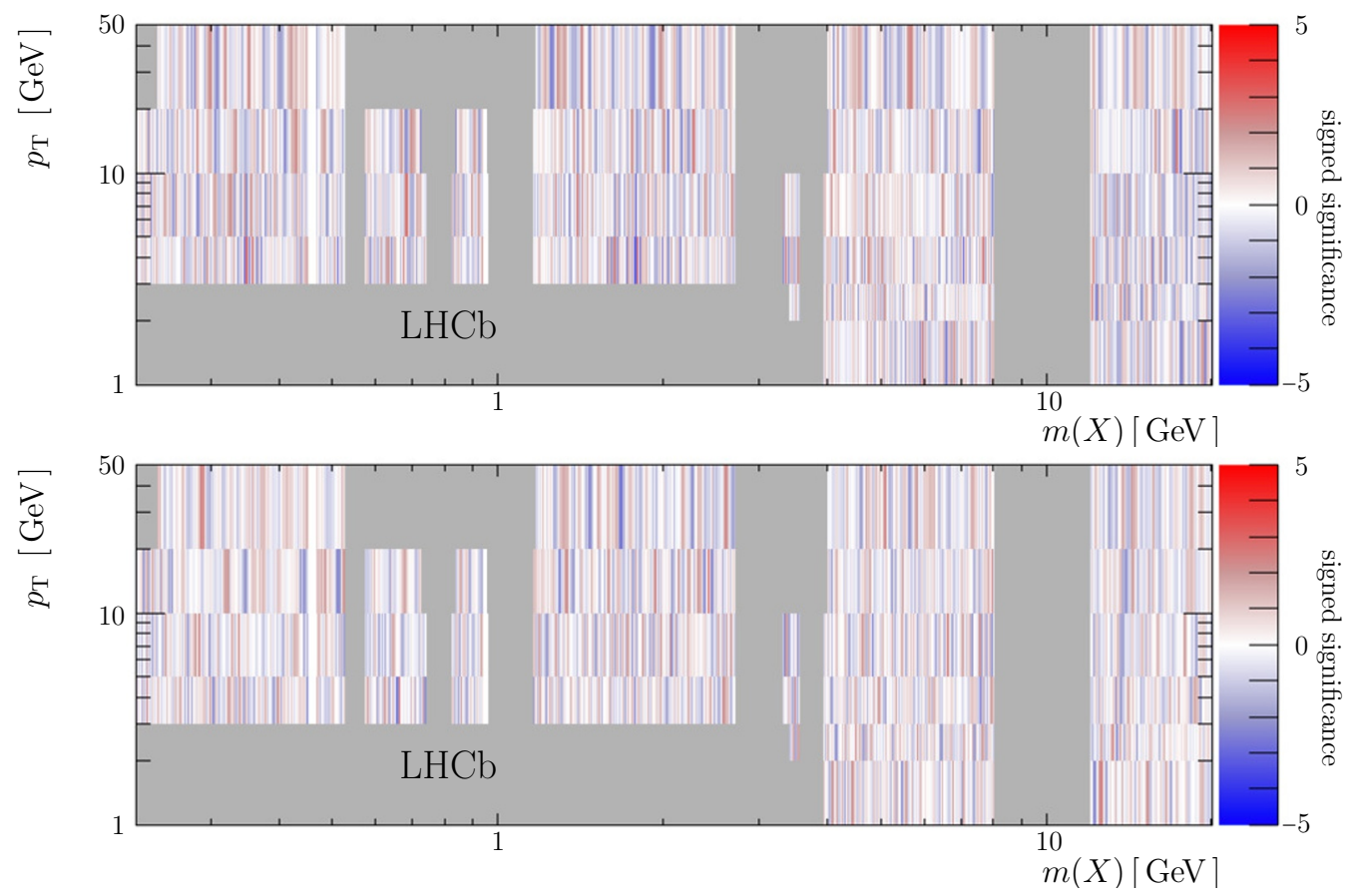

Figure 3. Signed local significances in the $m(X)<20 \mathrm{GeV}$ region for the (top) inclusive and (bottom) associated beauty searches for prompt $X \rightarrow \mu^{+} \mu^{-}$decays. If the best-fit signal-yield estimator is negative, the signed significance is negative and vice versa. The grey regions are excluded either due to a nearby large QCD resonance contribution, or because the overlap of the bin with the fiducial region in table 1 is small.

Motivated by the possible excess seen by CMS [49] in $X+b \bar{b}$ events, a dedicated search for a resonance with $27<m(X)<30 \mathrm{GeV}$ and $0.5<\Gamma(X)<3.0 \mathrm{GeV}$ is performed in the subset of the $X+b$ candidates that contains at least two $b$-tagged jets. The mass spectrum in the range $20-40 \mathrm{GeV}$ is fitted using a model consisting of a second-order polynomial background and a signal whose mass and width are free to vary within the $m(X)$ and $\Gamma(X)$ ranges specified above. Figure 5 shows the result of this fit. The best-fit signal yield is negative in the region considered; therefore, no evidence for a signal is observed. Using the efficiency and luminosity from section 5 , and their associated uncertainties, the upper limits on the $X\left(\mu^{+} \mu^{-}\right)+b \bar{b}$ cross section in the $m(X)$ and $\Gamma(X)$ regions considered are no larger than $15 \mathrm{fb} \times \sqrt{\Gamma(X) / \mathrm{GeV}}$.

The fit strategy used in the searches for displaced $X \rightarrow \mu^{+} \mu^{-}$decays below the $K_{\mathrm{S}}^{0}$ mass is also the same as in refs. [14, 15]. Binned extended maximum-likelihood fits are performed to the $Q$ spectrum in each $p_{\mathrm{T}}$ bin. The region near the $K_{\mathrm{S}}^{0}$ mass is vetoed to avoid the sizable background from doubly misidentified $K_{\mathrm{S}}^{0} \rightarrow \pi^{+} \pi^{-}$decays. The expected photon-conversion contribution is derived from a sample of candidates that are consistent with a photon originating from a PV. Two large control samples are used to develop and validate the modeling of the $K_{\mathrm{S}}^{0}$ and remaining material-interaction contributions: dimuon candidates that fail, but nearly satisfy, the stringent muon-identification criteria; and a sample of dimuon candidates that is rejected by the material-interaction criterion. 


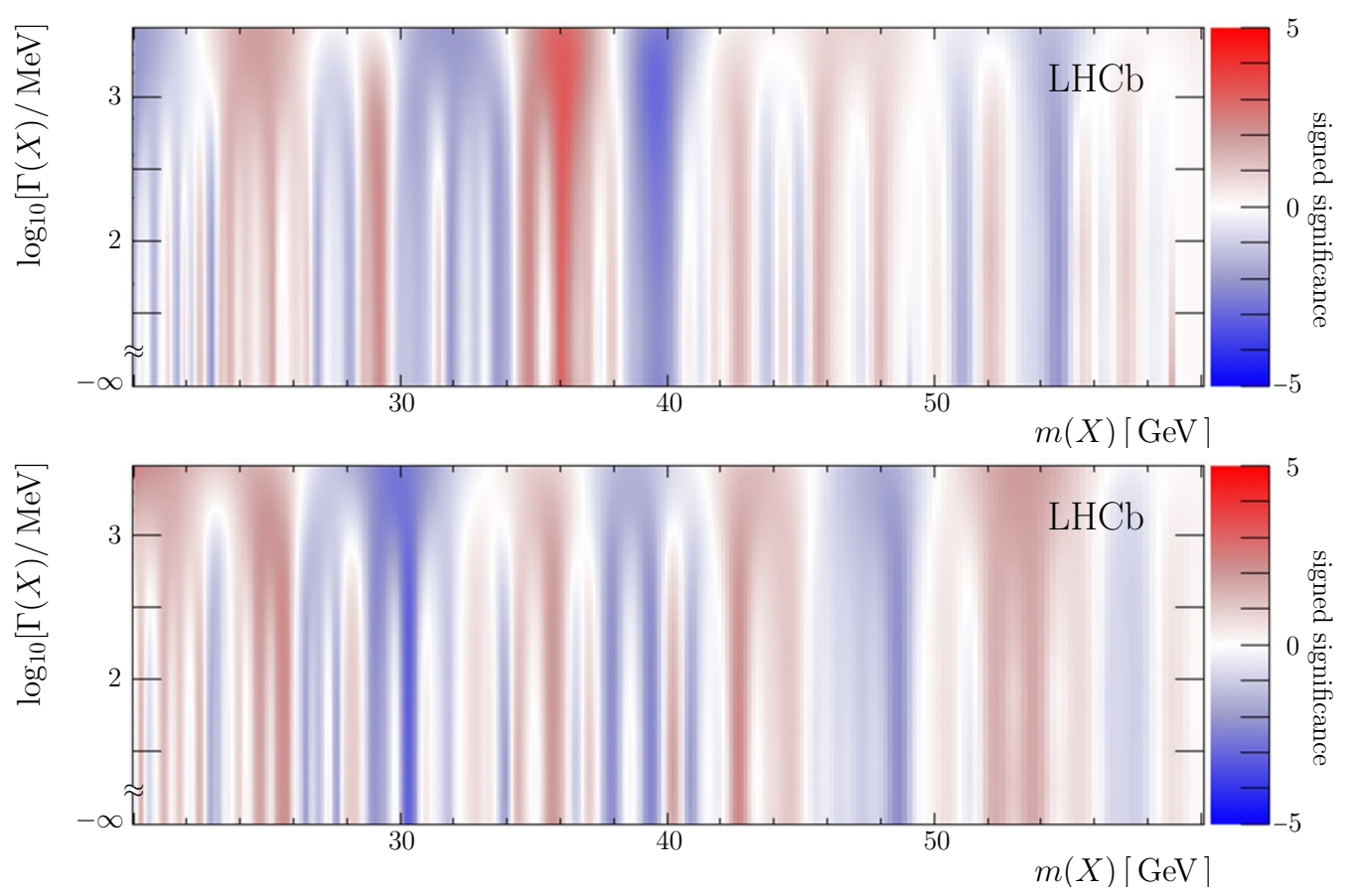

Figure 4. Signed local significances in the $m(X)>20 \mathrm{GeV}$ region for the (top) inclusive and (bottom) associated beauty searches for prompt $X \rightarrow \mu^{+} \mu^{-}$decays. The lower limit on the vertical axis of $\log _{10}[\Gamma(X) / \mathrm{MeV}]=-\infty$ corresponds to $\Gamma(X)=0$.

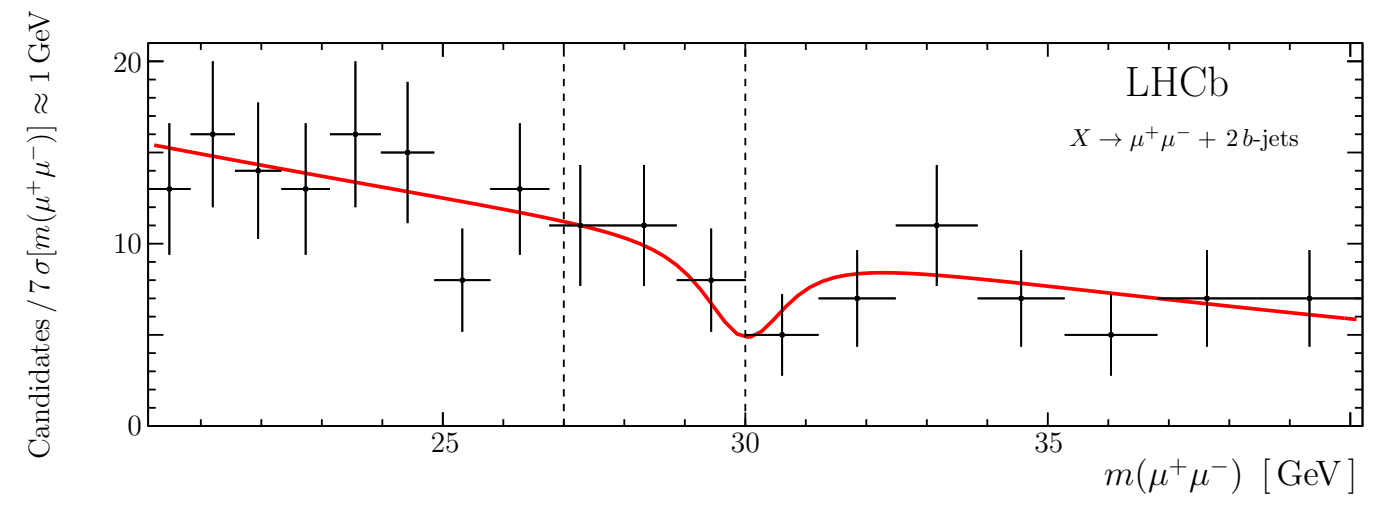

Figure 5. Fit to the $m\left(\mu^{+} \mu^{-}\right)$spectrum in events with at least two $b$-tagged jets. The $27<m(X)<30 \mathrm{GeV}$ search region is marked by the vertical dashed lines.

Both contributions are well modeled by second-order polynomials in $Q$ below the $K_{\mathrm{S}}^{0}$ veto region. The material-interaction contribution, apart from the dedicated photon-conversion component, is not needed in the search that requires a decay topology consistent with an $X$ boson originating from a $\mathrm{PV}$.

The fit strategy used in the searches for displaced $X \rightarrow \mu^{+} \mu^{-}$decays above the $K_{\mathrm{S}}^{0}$ veto region, specifically, in the $0.5<m(X)<3.0 \mathrm{GeV}$ mass range, is the same as used in the LHCb search for hidden-sector bosons produced in $B^{0} \rightarrow K^{(*)} X\left(\mu^{+} \mu^{-}\right)$decays [50, 51]. This strategy was first introduced in ref. [48]. Since no sharp features are expected in 

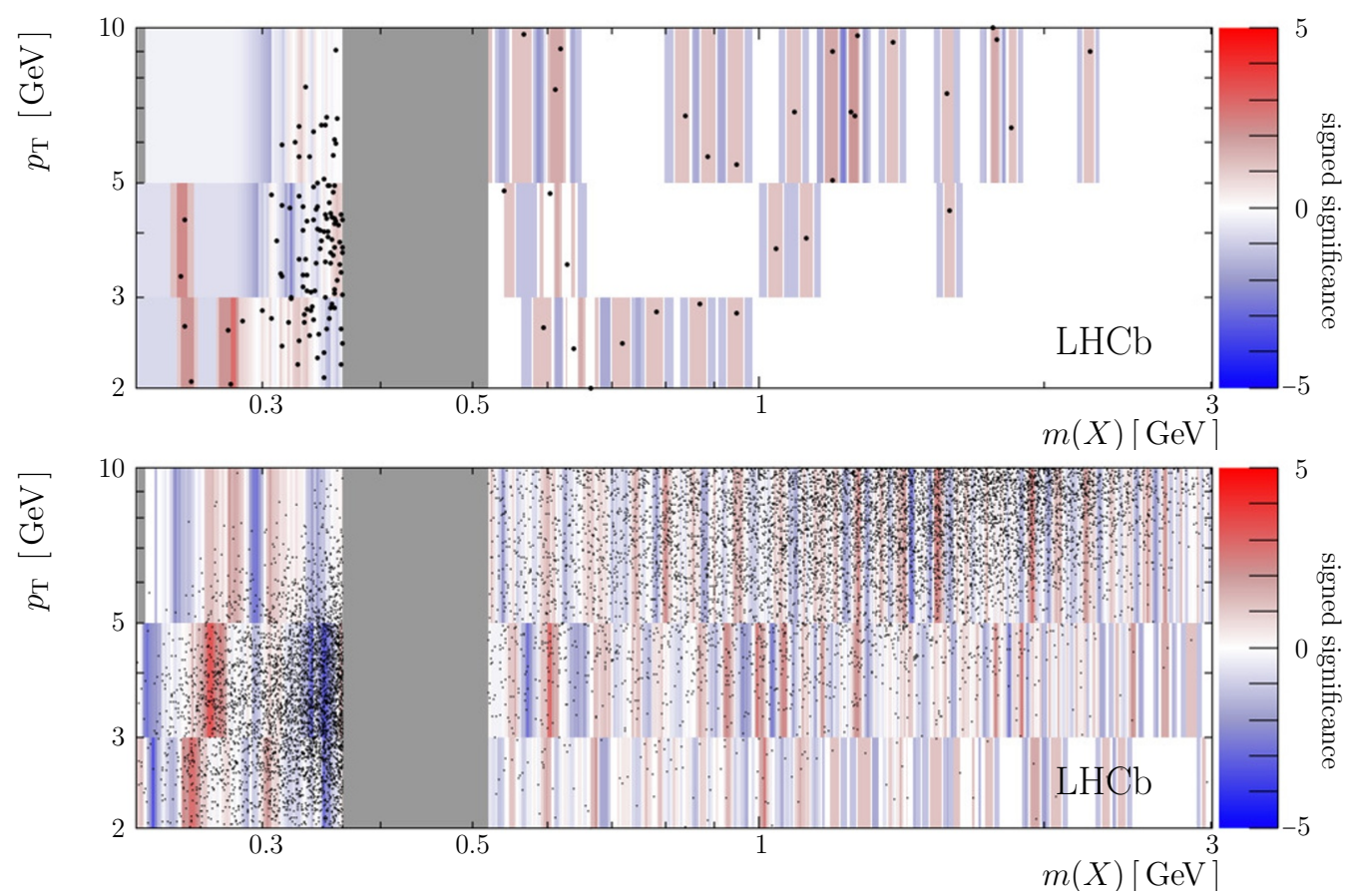

Figure 6. Signed local significances for the (top) promptly produced and (bottom) inclusive searches for displaced $X \rightarrow \mu^{+} \mu^{-}$decays. The black points show the individual candidates.

the background in this region, and due to the small bin occupancies, the background is estimated by interpolating the yields in the sidebands starting at $\pm 3 \sigma\left[m\left(\mu^{+} \mu^{-}\right)\right]$from $m(X)$. The statistical test at each mass is based on the profile likelihood ratio of Poissonprocess hypotheses with and without a signal contribution. The uncertainty on the linearity of the background interpolation is modeled by a Gaussian term in the likelihood.

Figure 6 shows the signed local significances for both searches for displaced $X \rightarrow \mu^{+} \mu^{-}$ decays. The largest local excess in the search for a promptly produced long-lived $X$ boson is $2.8 \sigma$, which occurs at $280 \mathrm{MeV}$ in the $2<p_{\mathrm{T}}(X)<3 \mathrm{GeV}$ bin. The largest local excess in the inclusive search for displaced $X \rightarrow \mu^{+} \mu^{-}$decays is $3.1 \sigma$ at $604 \mathrm{MeV}$ in the $3<p_{\mathrm{T}}(X)<5 \mathrm{GeV}$ bin. Both of these correspond to global excesses below $1 \sigma$; therefore, no significant excess is found in either search for displaced $X \rightarrow \mu^{+} \mu^{-}$decays.

\section{$5 \quad$ Efficiency and luminosity}

The $X \rightarrow \mu^{+} \mu^{-}$yields are corrected for detection efficiency, which is determined as the product of the trigger, reconstruction, and selection efficiencies. The hardware trigger efficiency is measured as a function of $\sqrt{p_{\mathrm{T}}\left(\mu^{+}\right) p_{\mathrm{T}}\left(\mu^{-}\right)}$using a displaced $J / \psi$ calibration sample. Events selected by the hardware trigger independently of the $J / \psi$ candidate, e.g. due to the presence of a high- $p_{\mathrm{T}}$ hadron, are used to determine the trigger efficiency directly from the data. The muon reconstruction efficiency is obtained from simulation in bins of $[p(\mu), \eta(\mu)]$. Scale factors that correct for discrepancies between the data and simulation are determined using a data-driven tag-and-probe approach on an independent 
sample of $J / \psi \rightarrow \mu^{+} \mu^{-}$decays [52]. The contribution to the selection efficiency from the muon-identification performance is measured in bins of $\left[p_{\mathrm{T}}(\mu), \eta(\mu)\right]$ using a highly pure calibration sample of $J / \psi \rightarrow \mu^{+} \mu^{-}$decays. Finally, the contributions from the vertexquality and prompt-decay criteria are determined from simulation, and validated using a calibration sample of prompt QCD resonance decays to the $\mu^{+} \mu^{-}$final state.

The uncertainty due to the methods used to determine each of these components of the total efficiency is assessed by repeating the data-based efficiency studies on simulated events, where the difference between the true and efficiency-corrected yields in kinematic bins is used to determine the systematic uncertainty. These uncertainties are in the $2-$ $5 \%$ range, depending on $X$-boson kinematics. Additional uncertainties arise due to the unknown production mechanisms of the $X$ bosons. The muon reconstruction and identification efficiencies depend on the charged-particle multiplicity. The corresponding systematic uncertainty is determined to be $5 \%$, which covers both minimal and maximal charged-particle multiplicities defined in table 1 at the $2 \sigma$ level. The unknown kinematic distributions in both $p_{\mathrm{T}}$ and $\eta$ within the wide $p_{\mathrm{T}}$ bins used in the analysis lead to sizable uncertainties. The variation in the efficiencies across the kinematic regions allowed in each bin are used to determine bin-dependent uncertainties that vary from 10 to $30 \%$.

The $X+b$ analysis uses the SV-based $b$-tagging method described in detail in ref. [42], though without placing any criteria on the boosted decision tree algorithms; only the presence of an SV is required. The $b$-tagging efficiency is estimated to be $(65 \pm 7) \%$, where the uncertainty covers both the variation of the $b$-tagging efficiency across the $b$ jet fiducial region and possible data-simulation discrepancies. An additional uncertainty arises since the efficiency for a $b$-tagged jet in the fiducial region to be reconstructed with $p_{\mathrm{T}}>20 \mathrm{GeV}$ depends on the unknown underlying jet $p_{\mathrm{T}}$ spectrum. The detector response to jets is studied using the $p_{\mathrm{T}}$-balance distribution of $p_{\mathrm{T}}(\mathrm{jet}) / p_{\mathrm{T}}(Z)$ in nearly back-to-back $Z$-boson+jet events using the same data-driven technique as in ref. [40]. Based on this study, and considering jet $p_{\mathrm{T}}$ spectra as soft as QCD di-b-jet production and hard enough to result in negligible inefficiency, this efficiency is estimated to be $(90 \pm 5) \%$.

The searches for displaced $X \rightarrow \mu^{+} \mu^{-}$decays must also account for effects that arise due to the displacement of the SV from the PV. The relative efficiency of displaced compared to prompt $X \rightarrow \mu^{+} \mu^{-}$decays is obtained as a function of $m(X)$ and $p_{\mathrm{T}}(X)$ by resampling prompt $X \rightarrow \mu^{+} \mu^{-}$decay candidates as displaced $X \rightarrow \mu^{+} \mu^{-}$decays, where all displacement-dependent properties are recalculated based on the resampled SV locations. The high-precision material map produced in ref. [43] forms the basis of the materialinteraction criterion applied in the selection. This map is used to determine where each muon would hit active sensors, and thus, have recorded hits in the VELO. The resolution on the vertex location and other displacement-dependent properties varies strongly with the location of the first VELO hit on each muon track, though this dependence is largely geometric, making rescaling the resolution of prompt tracks straightforward. This approach is validated using simulation, where prompt $X \rightarrow \mu^{+} \mu^{-}$decays are used to predict the properties of long-lived $X$ bosons; these predictions are found to agree within $2 \%$ with the actual values. The efficiencies at both short and long distances, which are driven by the muon displacement criterion and the minimum number of VELO hits required to form a track, 
respectively, are well described. The dominant uncertainty, which arises due to limited knowledge of how radiation damage has affected the VELO performance, is estimated to be $5 \%$ by rerunning the resampling method under different radiation-damage hypotheses.

The efficiency of the material-interaction criterion is validated separately using two control samples. The predicted efficiency for an $X$ boson with the same mass and lifetime as the $K_{\mathrm{S}}^{0}$ meson is compared to the efficiency observed in a control sample of $K_{\mathrm{S}}^{0}$ decays. The predicted and observed efficiencies agree to 1\%. Additionally, in ref. [43] the expected performance of the material-interaction criterion was shown to agree with the performance observed in a control sample of photon conversions to the $\mathcal{O}\left(10^{-4}\right)$ level. Finally, the distribution of the SV locations is unknown, which leads to a $10 \%$ uncertainty in the efficiency determined by comparing the efficiency of an $X$ boson that rarely survives long enough to enter the decay fiducial region to an extremely long-lived $X$ boson.

Most of the data used in this analysis is from data-taking periods that do not yet have fully calibrated luminosities. Therefore, the efficiency-corrected yield of $Z / \gamma^{*} \rightarrow \mu^{+} \mu^{-}$ decays observed in the data sample - and the corresponding high-precision LHCb crosssection measurement made using 2015 data [53] — are used to infer the luminosity. A small correction factor is obtained from PyTHIA 8 to account for the different fiducial regions. This luminosity determination is validated by also determining the $\Upsilon(1 S)$ differential cross section from this data sample and comparing the results to those published by LHCb using the 2015 data sample [54]. The different fiducial region is again corrected for using a scale factor obtained from PyтнiA 8 . The results are found to agree to $\approx 5 \%$ in each $p_{\mathrm{T}}$ bin, which is assigned as a systematic uncertainty and combined with the $4 \%$ luminosity uncertainty from ref. [53] to obtain the total uncertainty on the luminosity of this data sample. Based on both of these studies, the luminosity is determined to be $5.1 \pm 0.3 \mathrm{fb}^{-1}$. The minimal dark-photon search [15], which used the same data sample but did not require knowledge of the luminosity, quotes an uncalibrated luminosity value that is $7 \%$ larger. The efficiency corrections used to infer the luminosity are highly correlated to those used to correct the observed $X \rightarrow \mu^{+} \mu^{-}$yields, which is accounted for when determining the total normalization uncertainties.

\section{Cross-section results}

The upper limits on the signal yields obtained in section 4 are normalized using the efficiencies and luminosity described in section 5 . The systematic uncertainties on the signal yield, efficiency, and luminosity are included in the profile likelihood when determining the cross-section upper limits. These uncertainties are described in detail in sections 4 and 5 , and summarized in table 2 . The resulting upper limits at $90 \%$ confidence level on $\sigma\left(X \rightarrow \mu^{+} \mu^{-}\right)$for all searches are shown in figures $7-9 .^{1}$

The model-independent limits in figures $7-8$ can be used to place constraints on any model that would produce a promptly decaying low-mass dimuon resonance within the fiducial region of table 1. For example, models where a complex scalar singlet is added to the two-Higgs doublet (2HDM) potential often feature a light pseudoscalar boson that

\footnotetext{
${ }^{1}$ Numerical results can be found in the CDS entry for LHCb-PAPER-2020-013, see https://cds.cern.ch/ record/2722971.
} 


\begin{tabular}{|lc|}
\hline Source & Relative uncertainty \\
\hline Signal model & $5 \%$ \\
Background model & data driven, see section 4 \\
\hline Trigger, reconstruction, selection & $2-5 \%$ (bin dependent) \\
Charged-particle multiplicity & $5 \%$ \\
$X$ kinematics & $10-30 \%$ (bin dependent) \\
$b$-jet selection & $11 \%(X+b$ only) \\
SV selection & $5 \%$ (SV-based only) \\
$X$ SV distribution & $10 \%$ (SV-based only) \\
\hline Luminosity & $6 \%$ \\
\hline Total & $11-30 \%$ (bin dependent) \\
\hline
\end{tabular}

Table 2. Summary of systematic uncertainties. The luminosity and efficiency uncertainties are highly correlated, which is accounted for when obtaining the total uncertainties.
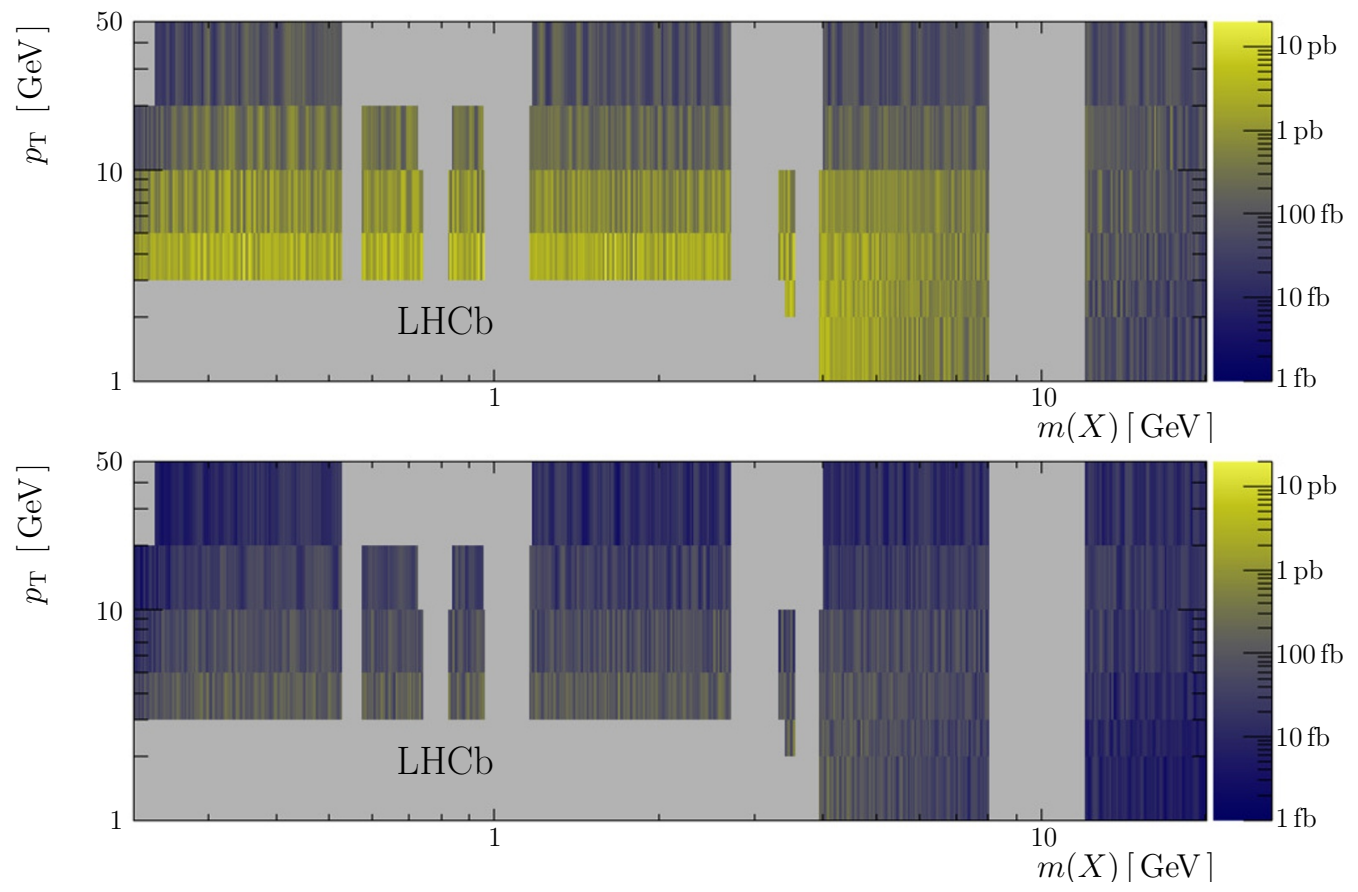

Figure 7. Upper limits at $90 \%$ confidence level on the cross section $\sigma\left(X \rightarrow \mu^{+} \mu^{-}\right)$in the $m(X)<20 \mathrm{GeV}$ region for the (top) inclusive and (bottom) associated beauty searches for prompt $X \rightarrow \mu^{+} \mu^{-}$decays. 

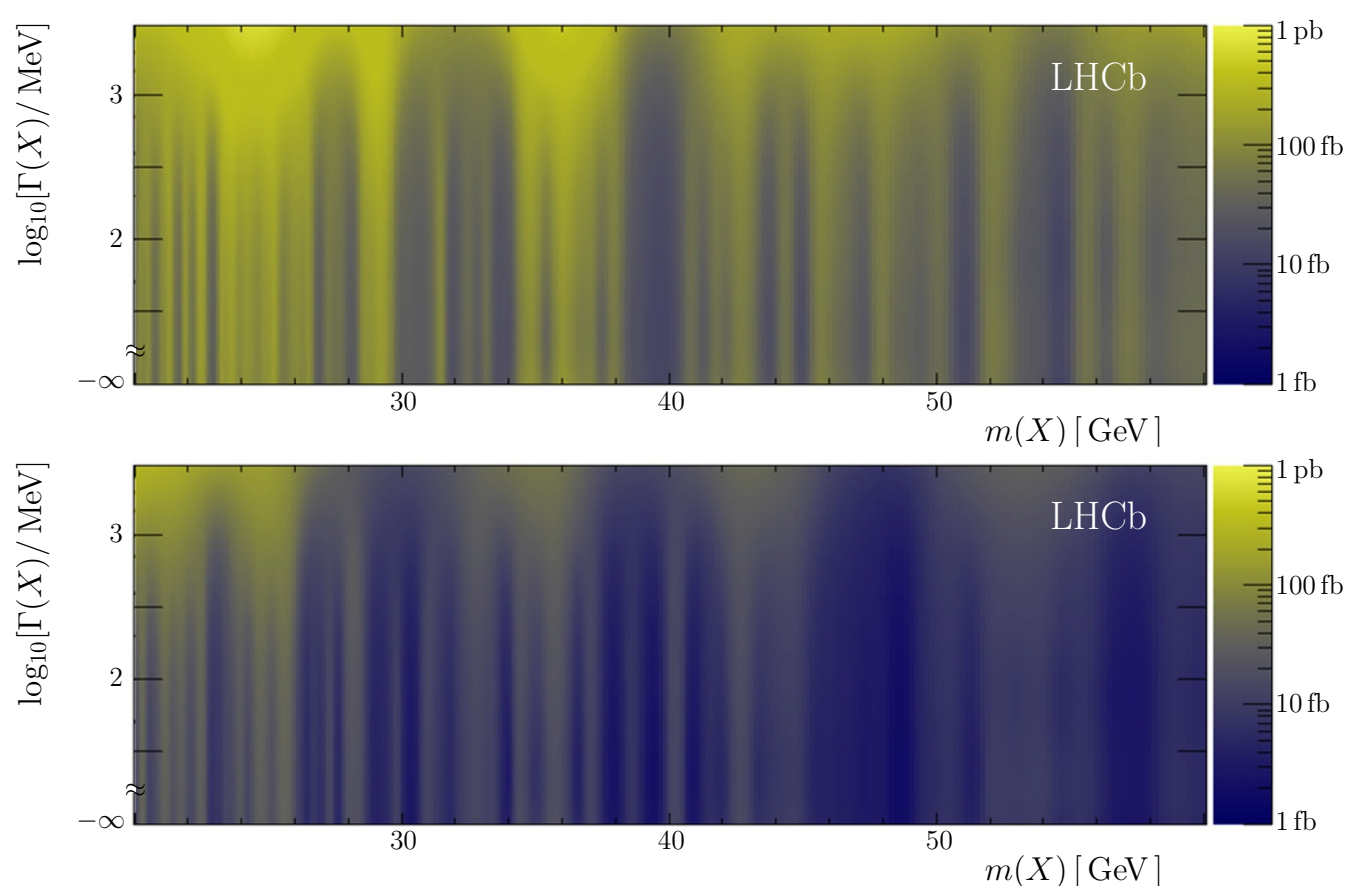

Figure 8. Upper limits at $90 \%$ confidence level on the cross section $\sigma\left(X \rightarrow \mu^{+} \mu^{-}\right)$in the $m(X)>20 \mathrm{GeV}$ region for the (top) inclusive and (bottom) associated beauty searches for prompt $X \rightarrow \mu^{+} \mu^{-}$decays.
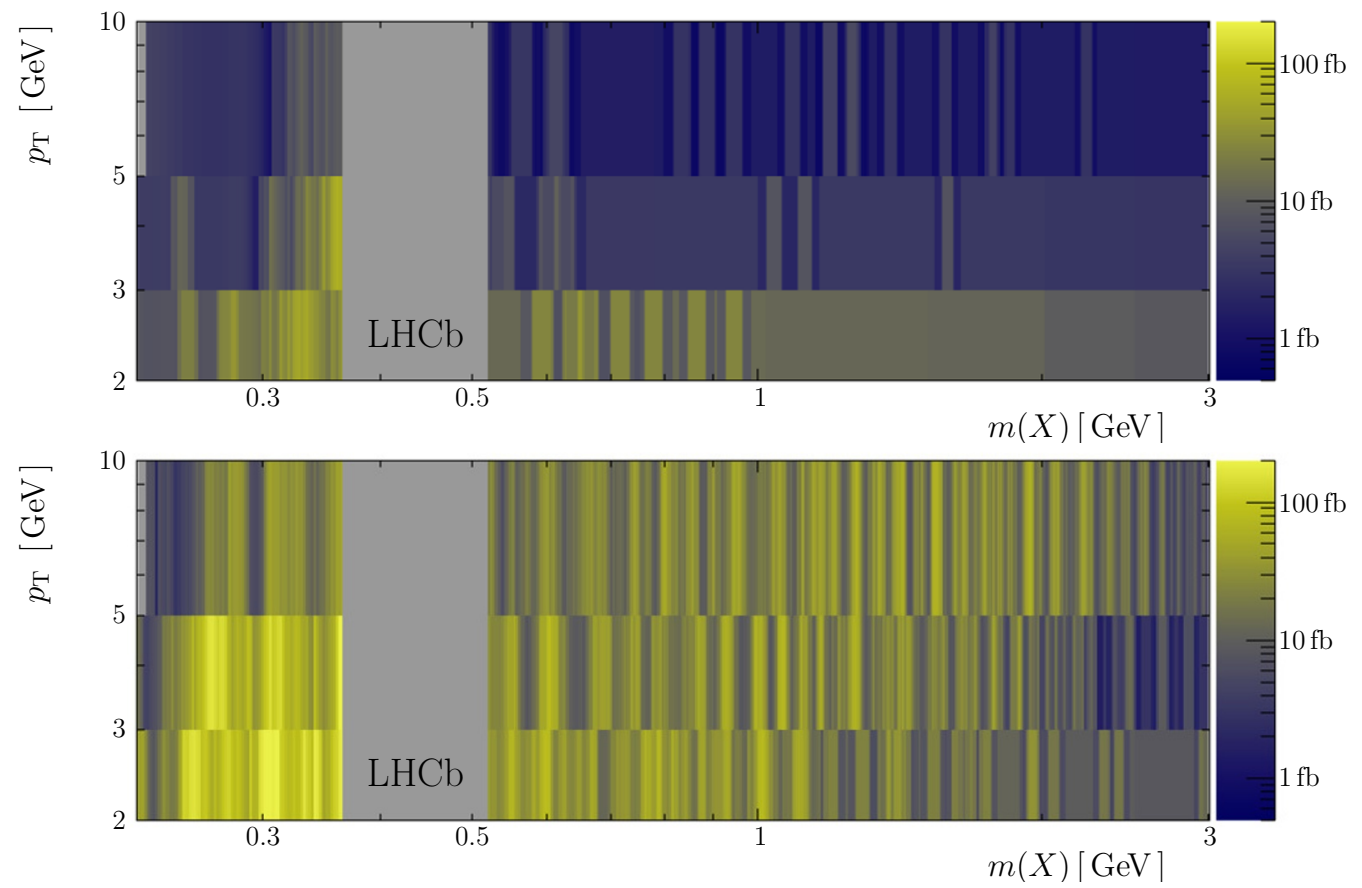

Figure 9. Upper limits at 90\% confidence level on the cross section $\sigma\left(X \rightarrow \mu^{+} \mu^{-}\right)$for the (top) promptly produced and (bottom) inclusive searches for displaced $X \rightarrow \mu^{+} \mu^{-}$decays. 


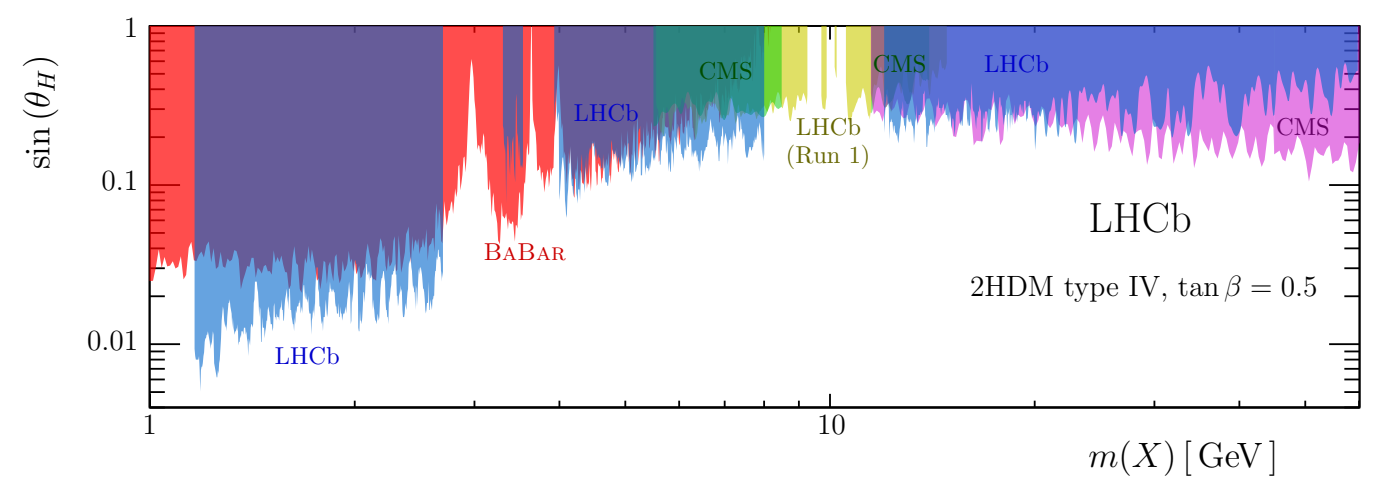

Figure 10. Upper limits at $90 \%$ confidence level on the $X-H$ mixing angle, $\theta_{H}$, for the $2 \mathrm{HDM}$ scenario discussed in the text (blue) from this analysis compared with existing limits from (red) BABAR [57], (green) CMS Run 1 [58], (magenta) CMS Run 2 [59] and (yellow) LHCb Run 1 [60].

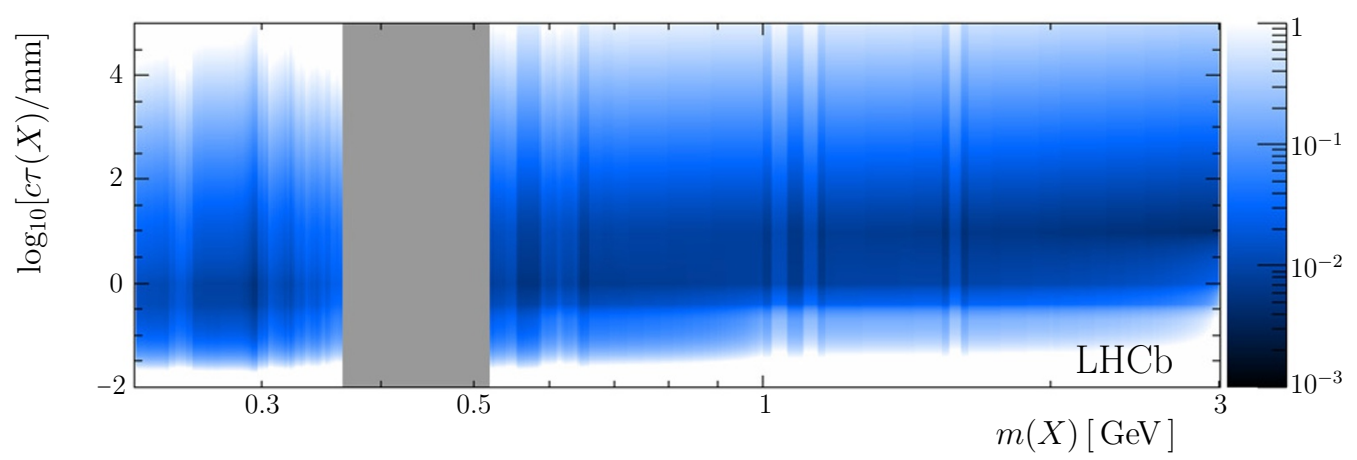

Figure 11. Upper limits at $90 \%$ confidence level on the $\gamma-Z_{\mathrm{HV}}$ kinetic mixing strength for the $\mathrm{HV}$ scenario discussed in the text. Here, $X$ denotes a composite HV vector boson.

can decay into the dimuon final state; see, e.g., ref. [18]. References [55, 56] considered the scenario where the pseudoscalar boson acquires all of its couplings to SM fermions through its mixing with the Higgs doublets; the corresponding $X-H$ mixing angle is denoted as $\theta_{H}$. Figure 10 shows that world-leading constraints are placed on $\theta_{H}$ by the $\sigma\left(X \rightarrow \mu^{+} \mu^{-}\right)$ limits shown in figures 7-8; these constraints are twice as strong in the $\mathcal{O}(\mathrm{GeV})$ region as those obtained by recasting the dark-photon results in ref. [15]. Furthermore, assuming the $X+b \bar{b}$ topology produced by this type of model permits direct comparison with the excess seen by CMS in this final state [49]. For this scenario, the $X+b$ limits from figure 8 are about 20 times lower than the excess observed by CMS.

The limits on displaced $X \rightarrow \mu^{+} \mu^{-}$decays in figure 9 can also be used to place constraints on specific models. One example is HV scenarios that exhibit confinement, which result in a large multiplicity of light hidden hadrons from showering processes [13]. These hidden hadrons typically have low $p_{\mathrm{T}}$ and decay displaced from the proton-proton collision. Figure 11 shows the limits placed on this type of HV scenario by the search for displaced $X \rightarrow \mu^{+} \mu^{-}$decays. These are the most stringent constraints to date. Specifically, constraints are placed on the kinetic-mixing strength between the photon and a heavy $\mathrm{HV}$ boson, $Z_{\mathrm{HV}}$, with photon-like couplings. The kinematics of the hidden hadrons depend 
upon the average $\mathrm{HV}$ hadron multiplicity, $\left\langle N_{\mathrm{HV}}\right\rangle$, and are largely independent of the model parameter space. In figure $11\left\langle N_{\mathrm{HV}}\right\rangle$ is fixed at $\approx 10$ for all hidden hadron masses. These are the first results that constrain the kinetic-mixing strength to be less than unity in this mass region.

\section{Summary}

In summary, searches are performed for low-mass dimuon resonances produced in protonproton collisions at a center-of-mass energy of $13 \mathrm{TeV}$ using a data sample corresponding to an integrated luminosity of $5.1 \mathrm{fb}^{-1}$ collected with the LHCb detector. The $X \rightarrow \mu^{+} \mu^{-}$ decays can be either prompt or displaced from the proton-proton collision, where in both cases the requirements placed on the event and the assumptions made about the production mechanisms are kept as minimal as possible. Two variations are performed of the search for prompt $X \rightarrow \mu^{+} \mu^{-}$decays: an inclusive version, and one where the $X$ boson is required to be produced in association with a beauty quark. Two variations are also considered of the search for displaced $X \rightarrow \mu^{+} \mu^{-}$decays: an inclusive version, and one where the $X$ boson is required to be produced promptly in the proton-proton collision. The searches for prompt $X \rightarrow \mu^{+} \mu^{-}$decays explore the mass range from near the dimuon threshold up to $60 \mathrm{GeV}$, with nonnegligible $X$ widths considered above $20 \mathrm{GeV}$. The searches for displaced $X \rightarrow \mu^{+} \mu^{-}$decays consider masses up to $3 \mathrm{GeV}$. None of the searches finds evidence for a signal, and $90 \%$ confidence-level exclusion limits are placed on the $X \rightarrow \mu^{+} \mu^{-}$cross sections, each with minimal model dependence.

\section{Acknowledgments}

We express our gratitude to our colleagues in the CERN accelerator departments for the excellent performance of the LHC. We thank the technical and administrative staff at the LHCb institutes. We acknowledge support from CERN and from the national agencies: CAPES, CNPq, FAPERJ and FINEP (Brazil); MOST and NSFC (China); CNRS/IN2P3 (France); BMBF, DFG and MPG (Germany); INFN (Italy); NWO (Netherlands); MNiSW and NCN (Poland); MEN/IFA (Romania); MSHE (Russia); MinECo (Spain); SNSF and SER (Switzerland); NASU (Ukraine); STFC (United Kingdom); DOE NP and NSF (U.S.A.). We acknowledge the computing resources that are provided by CERN, IN2P3 (France), KIT and DESY (Germany), INFN (Italy), SURF (Netherlands), PIC (Spain), GridPP (United Kingdom), RRCKI and Yandex LLC (Russia), CSCS (Switzerland), IFINHH (Romania), CBPF (Brazil), PL-GRID (Poland) and OSC (U.S.A.). We are indebted to the communities behind the multiple open-source software packages on which we depend. Individual groups or members have received support from AvH Foundation (Germany); EPLANET, Marie Skłodowska-Curie Actions and ERC (European Union); A*MIDEX, ANR, Labex P2IO and OCEVU, and Région Auvergne-Rhône-Alpes (France); Key Research Program of Frontier Sciences of CAS, CAS PIFI, and the Thousand Talents Program (China); RFBR, RSF and Yandex LLC (Russia); GVA, XuntaGal and GENCAT (Spain); the Royal Society and the Leverhulme Trust (United Kingdom). 
Open Access. This article is distributed under the terms of the Creative Commons Attribution License (CC-BY 4.0), which permits any use, distribution and reproduction in any medium, provided the original author(s) and source are credited.

\section{References}

[1] M. Battaglieri et al., US Cosmic Visions: New Ideas in Dark Matter 2017: Community Report, arXiv:1707.04591 [INSPIRE].

[2] P. Fayet, On the search for a new spin 1 boson, Nucl. Phys. B 187 (1981) 184 [InSPIRE].

[3] P. Fayet, Effects of the spin 1 partner of the goldstino (gravitino) on neutral current phenomenology, Phys. Lett. B 95 (1980) 285 [INSPIRE].

[4] L.B. Okun, Limits of electrodynamics: paraphotons?, Sov. Phys. JETP 56 (1982) 502 [INSPIRE].

[5] P. Galison and A. Manohar, Two Z's or not two Z's?, Phys. Lett. B 136 (1984) 279 [INSPIRE].

[6] B. Holdom, Two U(1)'s and $\epsilon$ charge shifts, Phys. Lett. B 166 (1986) 196 [InSPIRE].

[7] M. Pospelov, A. Ritz and M.B. Voloshin, Secluded WIMP dark matter, Phys. Lett. B 662 (2008) 53 [arXiv:0711.4866] [INSPIRE].

[8] N. Arkani-Hamed, D.P. Finkbeiner, T.R. Slatyer and N. Weiner, A theory of dark matter, Phys. Rev. D 79 (2009) 015014 [arXiv:0810.0713] [InSPIRE].

[9] J.D. Bjorken, R. Essig, P. Schuster and N. Toro, New fixed-target experiments to search for dark gauge forces, Phys. Rev. D 80 (2009) 075018 [arXiv:0906.0580] [INSPIRE].

[10] P. Ilten, Y. Soreq, M. Williams and W. Xue, Serendipity in dark photon searches, JHEP 06 (2018) 004 [arXiv : 1801.04847] [inSPIRE].

[11] P. Fayet, Extra U(1)'s and new forces, Nucl. Phys. B 347 (1990) 743 [InSPIRE].

[12] A. Berlin, N. Blinov, S. Gori, P. Schuster and N. Toro, Cosmology and accelerator tests of strongly interacting dark matter, Phys. Rev. D 97 (2018) 055033 [arXiv:1801.05805] [INSPIRE].

[13] A. Pierce, B. Shakya, Y. Tsai and Y. Zhao, Searching for confining hidden valleys at LHCb, ATLAS, and CMS, Phys. Rev. D 97 (2018) 095033 [arXiv:1708.05389] [InSPIRE].

[14] LHCb collaboration, Search for dark photons produced in $13 \mathrm{TeV}$ pp collisions, Phys. Rev. Lett. 120 (2018) 061801 [arXiv: 1710.02867] [INSPIRE].

[15] LHCb collaboration, Search for $A^{\prime} \rightarrow \mu^{+} \mu^{-}$decays, Phys. Rev. Lett. 124 (2020) 041801 [arXiv: 1910.06926] [INSPIRE].

[16] P. Ilten, J. Thaler, M. Williams and W. Xue, Dark photons from charm mesons at LHCb, Phys. Rev. D 92 (2015) 115017 [arXiv:1509.06765] [INSPIRE].

[17] P. Ilten, Y. Soreq, J. Thaler, M. Williams and W. Xue, Proposed inclusive dark photon search at LHCb, Phys. Rev. Lett. 116 (2016) 251803 [arXiv:1603.08926] [INSPIRE].

[18] G.C. Branco, P.M. Ferreira, L. Lavoura, M.N. Rebelo, M. Sher and J.P. Silva, Theory and phenomenology of two-Higgs-doublet models, Phys. Rept. 516 (2012) 1 [arXiv:1106.0034] [INSPIRE].

[19] M. Cacciari, G.P. Salam and G. Soyez, The anti- $k_{t}$ jet clustering algorithm, JHEP 04 (2008) 063 [arXiv: 0802 .1189] [INSPIRE].

[20] LHCb collaboration, The LHCb detector at the LHC, 2008 JINST 3 S08005 [INSPIRE]. 
[21] LHCb collaboration, LHCb detector performance, Int. J. Mod. Phys. A 30 (2015) 1530022 [arXiv: 1412.6352] [INSPIRE].

[22] G. Dujany and B. Storaci, Real-time alignment and calibration of the LHCb Detector in Run II, J. Phys. Conf. Ser. 664 (2015) 082010 [INSPIRE].

[23] R. Aaij et al., The LHCb trigger and its performance in 2011, 2013 JINST 8 P04022 [arXiv: 1211.3055] [INSPIRE].

[24] R. Aaij et al., Tesla: an application for real-time data analysis in High Energy Physics, Comput. Phys. Commun. 208 (2016) 35 [arXiv:1604.05596] [InSPIRE].

[25] T. Sjöstrand, S. Mrenna and P.Z. Skands, A brief introduction to PYTHIA 8.1, Comput. Phys. Commun. 178 (2008) 852 [arXiv:0710. 3820] [INSPIRE].

[26] T. Sjöstrand, S. Mrenna and P.Z. Skands, PYTHIA 6.4 physics and manual, JHEP 05 (2006) 026 [hep-ph/0603175] [INSPIRE].

[27] LHCb collaboration, Handling of the generation of primary events in Gauss, the LHCb simulation framework, J. Phys. Conf. Ser. 331 (2011) 032047 [inSPIRE].

[28] D.J. Lange, The EvtGen particle decay simulation package, Nucl. Instrum. Meth. A 462 (2001) 152 [INSPIRE].

[29] P. Golonka and Z. Was, PHOTOS Monte Carlo: a precision tool for QED corrections in Z and $W$ decays, Eur. Phys. J. C 45 (2006) 97 [hep-ph/0506026] [INSPIRE].

[30] Geant4 collaboration, Geant4 developments and applications, IEEE Trans. Nucl. Sci. 53 (2006) 270.

[31] Geant4 collaboration, Geant4 - a simulation toolkit, Nucl. Instrum. Meth. A 506 (2003) 250 [INSPIRE].

[32] LHCb collaboration, The LHCb simulation application, Gauss: Design, evolution and experience, J. Phys. Conf. Ser. 331 (2011) 032023 [InSPIRE].

[33] M. Spira, A. Djouadi, D. Graudenz and P.M. Zerwas, Higgs boson production at the LHC, Nucl. Phys. B 453 (1995) 17 [hep-ph/9504378] [INSPIRE].

[34] M. Spira, HIGLU: A program for the calculation of the total Higgs production cross-section at hadron colliders via gluon fusion including QCD corrections, hep-ph/9510347 [INSPIRE].

[35] NNPDF collaboration, Parton distributions for the LHC Run II, JHEP 04 (2015) 040 [arXiv: 1410.8849] [INSPIRE].

[36] A. Djouadi, J. Kalinowski and M. Spira, HDECAY: A Program for Higgs boson decays in the standard model and its supersymmetric extension, Comput. Phys. Commun. 108 (1998) 56 [hep-ph/9704448] [INSPIRE].

[37] A. Djouadi, J. Kalinowski, M. Muehlleitner and M. Spira, HDECAY: Twenty $y_{++}$years after, Comput. Phys. Commun. 238 (2019) 214 [arXiv:1801.09506] [INSPIRE].

[38] T. Sjöstrand et al., An introduction to PYTHIA 8.2, Comput. Phys. Commun. 191 (2015) 159 [arXiv: 1410.3012] [INSPIRE].

[39] P. Schwaller, D. Stolarski and A. Weiler, Emerging Jets, JHEP 05 (2015) 059 [arXiv: 1502.05409] [INSPIRE].

[40] LHCb collaboration, Study of forward $Z+$ jet production in $p p$ collisions at $\sqrt{s}=7 \mathrm{TeV}$, JHEP 01 (2014) 033 [arXiv:1310.8197] [INSPIRE].

[41] M. Cacciari, G.P. Salam and G. Soyez, FastJet user manual, Eur. Phys. J. C 72 (2012) 1896 [arXiv: 1111.6097] [INSPIRE]. 
[42] LHCb collaboration, Identification of beauty and charm quark jets at LHCb, 2015 JINST 10 P06013 [arXiv: 1504.07670] [INSPIRE].

[43] M. Alexander et al., Mapping the material in the LHCb vertex locator using secondary hadronic interactions, 2018 JINST 13 P06008 [arXiv:1803.07466] [INSPIRE].

[44] LHCb collaboration, Improved limit on the branching fraction of the rare decay $K_{\mathrm{S}}^{0} \rightarrow \mu^{+} \mu^{-}$, Eur. Phys. J. C 77 (2017) 678 [arXiv:1706.00758] [InSPIRE].

[45] W.A. Rolke, A.M. Lopez and J. Conrad, Limits and confidence intervals in the presence of nuisance parameters, Nucl. Instrum. Meth. A 551 (2005) 493 [physics/0403059] [InSPIRE].

[46] M. Williams, A novel approach to the bias-variance problem in bump hunting, 2017 JINST 12 P09034 [arXiv: 1705. 03578] [inSPIRE].

[47] P.D. Dauncey, M. Kenzie, N. Wardle and G.J. Davies, Handling uncertainties in background shapes: the discrete profiling method, 2015 JINST 10 P04015 [arXiv: 1408.6865] [INSPIRE].

[48] M. Williams, Searching for a particle of unknown mass and lifetime in the presence of an unknown non-monotonic background, 2015 JINST 10 P06002 [arXiv:1503.04767] [INSPIRE].

[49] CMS collaboration, Search for resonances in the mass spectrum of muon pairs produced in association with $b$ quark jets in proton-proton collisions at $\sqrt{s}=8$ and 13 TeV, JHEP 11 (2018) 161 [arXiv: 1808.01890] [INSPIRE].

[50] LHCb collaboration, Search for hidden-sector bosons in $B^{0} \rightarrow K^{* 0} \mu^{+} \mu^{-}$decays, Phys. Rev. Lett. 115 (2015) 161802 [arXiv: 1508.04094] [INSPIRE].

[51] LHCb collaboration, Search for long-lived scalar particles in $B^{+} \rightarrow K^{+} \chi\left(\mu^{+} \mu^{-}\right)$decays, Phys. Rev. D 95 (2017) 071101 [arXiv:1612.07818] [INSPIRE].

[52] LHCb collaboration, Measurement of the track reconstruction efficiency at LHCb, 2015 JINST 10 P02007 [arXiv:1408.1251] [INSPIRE].

[53] LHCb collaboration, Measurement of the forward $Z$ boson production cross-section in $p p$ collisions at $\sqrt{s}=13 \mathrm{TeV}$, JHEP 09 (2016) 136 [arXiv: 1607.06495] [INSPIRE].

[54] LHCb collaboration, Measurement of $\Upsilon$ production in pp collisions at $\sqrt{s}=13 \mathrm{TeV}$, JHEP 07 (2018) 134 [Erratum ibid. 05 (2019) 076] [arXiv: 1804.09214] [INSPIRE].

[55] U. Haisch and J.F. Kamenik, Searching for new spin-0 resonances at LHCb, Phys. Rev. D 93 (2016) 055047 [arXiv:1601.05110] [InSPIRE].

[56] U. Haisch, J.F. Kamenik, A. Malinauskas and M. Spira, Collider constraints on light pseudoscalars, JHEP 03 (2018) 178 [arXiv:1802.02156] [INSPIRE].

[57] BABAR collaboration, Search for di-muon decays of a low-mass Higgs boson in radiative decays of the $\Upsilon(1 S)$, Phys. Rev. D 87 (2013) 031102 [Erratum ibid. 87 (2013) 059903] [arXiv: 1210.0287] [INSPIRE].

[58] CMS collaboration, Search for a light pseudoscalar Higgs boson in the dimuon decay channel in pp collisions at $\sqrt{s}=7$ TeV, Phys. Rev. Lett. 109 (2012) 121801 [arXiv:1206.6326] [INSPIRE].

[59] CMS collaboration, Search for a narrow resonance lighter than $200 \mathrm{GeV}$ decaying to a pair of muons in proton-proton collisions at $\sqrt{s}=13 \mathrm{TeV}$, Phys. Rev. Lett. 124 (2020) 131802 [arXiv: 1912.04776] [INSPIRE].

[60] LHCb collaboration, Search for a dimuon resonance in the $\Upsilon$ mass region, JHEP 09 (2018) 147 [arXiv: 1805.09820] [INSPIRE]. 


\section{The LHCb collaboration}

R. Aaij ${ }^{31}$, C. Abellán Beteta ${ }^{49}$, T. Ackernley ${ }^{59}$, B. Adeva ${ }^{45}$, M. Adinolfi ${ }^{53}$, H. Afsharnia ${ }^{9}$, C.A. Aidala ${ }^{82}$, S. Aiola ${ }^{25}$, Z. Ajaltouni ${ }^{9}$, S. Akar ${ }^{64}$, J. Albrecht ${ }^{14}$, F. Alessio ${ }^{47}$, M. Alexander ${ }^{58}$, A. Alfonso Albero ${ }^{44}$, Z. Aliouche ${ }^{61}$, G. Alkhazov ${ }^{37}$, P. Alvarez Cartelle ${ }^{47}$, A.A. Alves Jr ${ }^{45}$, S. Amato ${ }^{2}$, Y. Amhis ${ }^{11}$, L. An ${ }^{21}$, L. Anderlini ${ }^{21}$, G. Andreassi ${ }^{48}$, A. Andreianov ${ }^{37}$, M. Andreotti ${ }^{20}$, F. Archilli ${ }^{16}$, A. Artamonov ${ }^{43}$, M. Artuso ${ }^{67}$, K. Arzymatov ${ }^{41}$, E. Aslanides ${ }^{10}$, M. Atzeni ${ }^{49}$, B. Audurier ${ }^{11}$, S. Bachmann ${ }^{16}$, M. Bachmayer ${ }^{48}$, J.J. Back ${ }^{55}$, S. Baker ${ }^{60}$, P. Baladron Rodriguez ${ }^{45}$, V. Balagura ${ }^{11, b}$, W. Baldini ${ }^{20}$, J. Baptista Leite ${ }^{1}$, R.J. Barlow ${ }^{61}$, S. Barsuk ${ }^{11}$, W. Barter ${ }^{60}$, M. Bartolini ${ }^{23,47, h}$, F. Baryshnikov ${ }^{79}$, J.M. Basels ${ }^{13}$, G. Bassi ${ }^{28}$, V. Batozskaya ${ }^{35}$, B. Batsukh ${ }^{67}$, A. Battig ${ }^{14}$, A. Bay ${ }^{48}$, M. Becker ${ }^{14}$, F. Bedeschi ${ }^{28}$, I. Bediaga ${ }^{1}$, A. Beiter ${ }^{67}$, V. Belavin ${ }^{41}$, S. Belin ${ }^{26}$, V. Bellee ${ }^{48}$, K. Belous ${ }^{43}$, I. Belyaev ${ }^{38}$, G. Bencivenni ${ }^{22}$, E. Ben-Haim ${ }^{12}$, A. Berezhnoy ${ }^{39}$, R. Bernet ${ }^{49}$, D. Berninghoff ${ }^{16}$, H.C. Bernstein ${ }^{67}$, C. Bertella ${ }^{47}$, E. Bertholet ${ }^{12}$, A. Bertolin ${ }^{27}$, C. Betancourt ${ }^{49}$, F. Betti ${ }^{19, e}$, M.O. Bettler ${ }^{54}$, Ia. Bezshyiko ${ }^{49}$, S. Bhasin ${ }^{53}$, J. Bhom ${ }^{33}$, L. Bian ${ }^{72}$, M.S. Bieker ${ }^{14}$, S. Bifani ${ }^{52}$, P. Billoir ${ }^{12}$, M. Birch ${ }^{60}$, F.C.R. Bishop ${ }^{54}$, A. Bizzeti ${ }^{21, t}$, M. Bjørn ${ }^{62}$, M.P. Blago ${ }^{47}$, T. Blake ${ }^{55}$, F. Blanc ${ }^{48}$, S. Blusk ${ }^{67}$, D. Bobulska ${ }^{58}$, V. Bocci ${ }^{30}$, J.A. Boelhauve ${ }^{14}$, O. Boente Garcia ${ }^{45}$, T. Boettcher ${ }^{63}$, A. Boldyrev ${ }^{80}$, A. Bondar ${ }^{42, w}$, N. Bondar ${ }^{37,47}$, S. Borghi ${ }^{61}$, M. Borisyak $^{41}$, M. Borsato ${ }^{16}$, J.T. Borsuk ${ }^{33}$, S.A. Bouchiba ${ }^{48}$, T.J.V. Bowcock ${ }^{59}$, A. Boyer ${ }^{47}$, C. Bozzi ${ }^{20}$, M.J. Bradley ${ }^{60}$, S. Braun ${ }^{65}$, A. Brea Rodriguez ${ }^{45}$, M. Brodski ${ }^{47}$, J. Brodzicka ${ }^{33}$, A. Brossa Gonzalo ${ }^{55}$, D. Brundu ${ }^{26}$, A. Buonaura ${ }^{49}$, C. Burr ${ }^{47}$, A. Bursche ${ }^{26}$, A. Butkevich ${ }^{40}$, J.S. Butter ${ }^{31}$, J. Buytaert ${ }^{47}$, W. Byczynski ${ }^{47}$, S. Cadeddu ${ }^{26}$, H. Cai ${ }^{72}$, R. Calabrese ${ }^{20, g}$, L. Calero Diaz ${ }^{22}$, S. Cali ${ }^{22}$, R. Calladine ${ }^{52}$, M. Calvi ${ }^{24, i}$, M. Calvo Gomez ${ }^{44, l}$, P. Camargo Magalhaes ${ }^{53}$, A. Camboni ${ }^{44}$, P. Campana ${ }^{22}$, D.H. Campora Perez ${ }^{47}$, A.F. Campoverde Quezada ${ }^{5}$, S. Capelli ${ }^{24, i}$, L. Capriotti ${ }^{19, e}$, A. Carbone ${ }^{19, e}$, G. Carboni ${ }^{29}$, R. Cardinale ${ }^{23, h}$, A. Cardini ${ }^{26}$, I. Carli ${ }^{6}$, P. Carniti ${ }^{24,}$, K. Carvalho Akiba ${ }^{31}$, A. Casais Vidal ${ }^{45}$, G. Casse ${ }^{59}$, M. Cattaneo ${ }^{47}$, G. Cavallero ${ }^{47}$, S. Celani ${ }^{48}$, R. Cenci ${ }^{28}$, J. Cerasoli ${ }^{10}$, A.J. Chadwick ${ }^{59}$, M.G. Chapman ${ }^{53}$, M. Charles $^{12}$, Ph. Charpentier ${ }^{47}$, G. Chatzikonstantinidis ${ }^{52}$, M. Chefdeville ${ }^{8}$, C. Chen ${ }^{3}$, S. Chen $^{26}$, A. Chernov ${ }^{33}$, S.-G. Chitic ${ }^{47}$, V. Chobanova ${ }^{45}$, S. Cholak $^{48}$, M. Chrzaszcz ${ }^{33}$, A. Chubykin ${ }^{37}$, V. Chulikov ${ }^{37}$, P. Ciambrone ${ }^{22}$, M.F. Cicala ${ }^{55}$, X. Cid Vidal ${ }^{45}$, G. Ciezarek ${ }^{47}$, F. Cindolo $^{19}$, P.E.L. Clarke ${ }^{57}$, M. Clemencic ${ }^{47}$, H.V. Cliff ${ }^{54}$, J. Closier ${ }^{47}$, J.L. Cobbledick ${ }^{61}$, V. Coco ${ }^{47}$, J.A.B. Coelho ${ }^{11}$, J. Cogan ${ }^{10}$, E. Cogneras ${ }^{9}$, L. Cojocariu ${ }^{36}$, P. Collins ${ }^{47}$, T. Colombo ${ }^{47}$, A. Contu ${ }^{26}$, N. Cooke ${ }^{52}$, G. Coombs ${ }^{58}$, S. Coquereau ${ }^{44}$, G. Corti ${ }^{47}$, C.M. Costa Sobral ${ }^{55}$, B. Couturier ${ }^{47}$, D.C. Craik ${ }^{63}$, J. Crkovská66 ${ }^{6}$, M. Cruz Torres ${ }^{1, y}$, R. Currie ${ }^{57}$, C.L. Da Silva ${ }^{66}$, E. Dall'Occo ${ }^{14}$, J. Dalseno ${ }^{45}$, C. D’Ambrosio ${ }^{47}$, A. Danilina ${ }^{38}$, P. d'Argent ${ }^{47}$, A. Davis ${ }^{61}$, O. De Aguiar Francisco ${ }^{47}$, K. De Bruyn ${ }^{47}$, S. De Capua ${ }^{61}$, M. De Cian ${ }^{48}$, J.M. De Miranda ${ }^{1}$, L. De Paula ${ }^{2}$, M. De Serio ${ }^{18, d}$, D. De Simone ${ }^{49}$, P. De Simone ${ }^{22}$, J.A. de Vries ${ }^{77}$, C.T. Dean ${ }^{66}$, W. Dean ${ }^{82}$, D. Decamp ${ }^{8}$, L. Del Buono ${ }^{12}$, B. Delaney ${ }^{54}$, H.-P. Dembinski ${ }^{14}$, A. Dendek ${ }^{34}$, X. Denis ${ }^{72}$, V. Denysenko ${ }^{49}$, D. Derkach ${ }^{80}$, O. Deschamps ${ }^{9}$, F. Desse ${ }^{11}$, F. Dettori ${ }^{26, f}$, B. Dey ${ }^{7}$, P. Di Nezza ${ }^{22}$, S. Didenko ${ }^{79}$, H. Dijkstra ${ }^{47}$, V. Dobishuk ${ }^{51}$, A.M. Donohoe ${ }^{17}$, F. Dordei ${ }^{26}$, M. Dorigo ${ }^{28, x}$, A.C. dos Reis ${ }^{1}$, L. Douglas ${ }^{58}$, A. Dovbnya ${ }^{50}$, A.G. Downes ${ }^{8}$, K. Dreimanis ${ }^{59}$, M.W. Dudek ${ }^{33}$, L. Dufour ${ }^{47}$, P. Durante ${ }^{47}$, J.M. Durham ${ }^{66}$, D. Dutta ${ }^{61}$, M. Dziewiecki ${ }^{16}$, A. Dziurda ${ }^{33}$, A. Dzyuba ${ }^{37}$, S. Easo ${ }^{56}$, U. Egede ${ }^{69}$, V. Egorychev ${ }^{38}$, S. Eidelman $^{42, w}$, S. Eisenhardt ${ }^{57}$, S. Ek-In ${ }^{48}$, L. Eklund ${ }^{58}$, S. Ely ${ }^{67}$, A. Ene ${ }^{36}$, E. Epple ${ }^{66}$, S. Escher ${ }^{13}$, J. Eschle ${ }^{49}$, S. Esen ${ }^{31}$, T. Evans ${ }^{47}$, A. Falabella ${ }^{19}$, J. Fan ${ }^{3}$, Y. Fan ${ }^{5}$, B. Fang ${ }^{72}$, N. Farley ${ }^{52}$, S. Farry ${ }^{59}$, D. Fazzini ${ }^{11}$, P. Fedin ${ }^{38}$, M. Féo ${ }^{47}$, P. Fernandez Declara ${ }^{47}$, A. Fernandez Prieto ${ }^{45}$, F. Ferrari ${ }^{19, e}$, L. Ferreira Lopes $^{48}$, F. Ferreira Rodrigues ${ }^{2}$, S. Ferreres Sole ${ }^{31}$, M. Ferrillo ${ }^{49}$, M. Ferro-Luzzi ${ }^{47}$, S. Filippov ${ }^{40}$, R.A. Fini ${ }^{18}$, M. Fiorini ${ }^{20, g}$, M. Firlej ${ }^{34}$, K.M. Fischer ${ }^{62}$, C. Fitzpatrick ${ }^{61}$, 
T. Fiutowski ${ }^{34}$, F. Fleuret ${ }^{11, b}$, M. Fontana ${ }^{47}$, F. Fontanelli ${ }^{23, h}$, R. Forty ${ }^{47}$, V. Franco Lima ${ }^{59}$, M. Franco Sevilla ${ }^{65}$, M. Frank ${ }^{47}$, E. Franzoso ${ }^{20}$, G. Frau $^{16}$, C. Frei ${ }^{47}$, D.A. Friday ${ }^{58}$, J. Fu ${ }^{25, p}$, Q. Fuehring ${ }^{14}$, W. Funk ${ }^{47}$, E. Gabriel ${ }^{31}$, T. Gaintseva ${ }^{41}$, A. Gallas Torreira ${ }^{45}$, D. Galli ${ }^{19, e}$, S. Gallorini ${ }^{27}$, S. Gambetta ${ }^{57}$, Y. Gan ${ }^{3}$, M. Gandelman ${ }^{2}$, P. Gandini ${ }^{25}$, Y. Gao ${ }^{4}$, M. Garau ${ }^{26}$, L.M. Garcia Martin ${ }^{46}$, P. Garcia Moreno ${ }^{44}$, J. García Pardiñas ${ }^{49}$, B. Garcia Plana ${ }^{45}$, F.A. Garcia Rosales ${ }^{11}$, L. Garrido ${ }^{44}$, D. Gascon ${ }^{44}$, C. Gaspar ${ }^{47}$, R.E. Geertsema ${ }^{31}$, D. Gerick ${ }^{16}$, L.L. Gerken ${ }^{14}$, E. Gersabeck ${ }^{61}$, M. Gersabeck ${ }^{61}$, T. Gershon ${ }^{55}$, D. Gerstel ${ }^{10}$, Ph. Ghez $^{8}$, V. Gibson ${ }^{54}$, A. Gioventù ${ }^{45}$, P. Gironella Gironell ${ }^{44}$, L. Giubega ${ }^{36}$, C. Giugliano ${ }^{20, g}$, K. Gizdov ${ }^{57}$, V.V. Gligorov ${ }^{12}$, C. Göbel ${ }^{70}$, E. Golobardes ${ }^{44, l}$, D. Golubkov ${ }^{38}$, A. Golutvin ${ }^{60,79}$, A. Gomes ${ }^{1, a}$, S. GOMEZ FERNANDEZ ${ }^{44}$, M. Goncerz ${ }^{33}$, P. Gorbounov ${ }^{38}$, I.V. Gorelov ${ }^{39}$, C. Gotti ${ }^{24, i}$, E. Govorkova ${ }^{31}$, J.P. Grabowski ${ }^{16}$, R. Graciani Diaz ${ }^{44}$, T. Grammatico ${ }^{12}$, L.A. Granado Cardoso ${ }^{47}$, E. Graugés ${ }^{44}$, E. Graverinii ${ }^{48}$, G. Graziani2 ${ }^{21}$, A. Grecu ${ }^{36}$, L.M. Greeven ${ }^{31}$, P. Griffith ${ }^{20}$, L. Grillo ${ }^{61}$, L. Gruber ${ }^{47}$, B.R. Gruberg Cazon ${ }^{62}$, C. Gu ${ }^{3}$, M. Guarise ${ }^{20}$, P. A. Günther ${ }^{16}$, E. Gushchin ${ }^{40}$, A. Guth ${ }^{13}$, Yu. Guz ${ }^{43,47}$, T. Gys ${ }^{47}$,

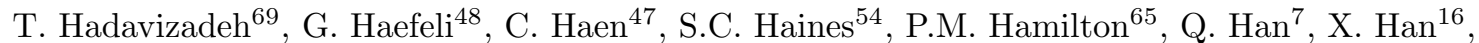

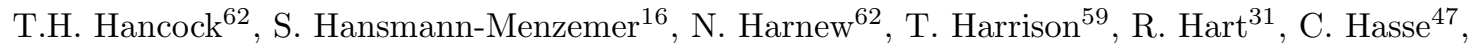
M. Hatch ${ }^{47}$, J. He ${ }^{5}$, M. Hecker ${ }^{60}$, K. Heijhoff ${ }^{31}$, K. Heinicke ${ }^{14}$, A.M. Hennequin ${ }^{47}$, K. Hennessy ${ }^{59}$, L. Henry ${ }^{25,46}$, J. Heuel ${ }^{13}$, A. Hicheur ${ }^{68}$, D. Hill ${ }^{62}$, M. Hilton ${ }^{61}$, S.E. Hollitt ${ }^{14}$, P.H. Hopchev ${ }^{48}$, J. $\mathrm{Hu}^{16}$, J. $\mathrm{Hu}^{71}$, W. $\mathrm{Hu}^{7}$, W. Huang ${ }^{5}$, W. Hulsbergen ${ }^{31}$, R.J. Hunter ${ }^{55}$, M. Hushchyn ${ }^{80}$,

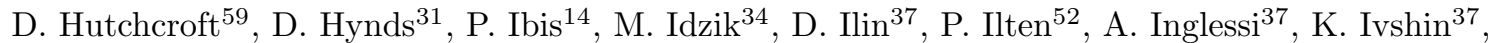
R. Jacobsson ${ }^{47}$, S. Jakobsen ${ }^{47}$, E. Jans ${ }^{31}$, B.K. Jashal ${ }^{46}$, A. Jawahery ${ }^{65}$, V. Jevtic ${ }^{14}$, F. Jiang ${ }^{3}$, M. John ${ }^{62}$, D. Johnson ${ }^{47}$, C.R. Jones ${ }^{54}$, T.P. Jones ${ }^{55}$, B. Jost ${ }^{47}$, N. Jurik ${ }^{62}$, S. Kandybei ${ }^{50}$, Y. Kang ${ }^{3}$, M. Karacson ${ }^{47}$, J.M. Kariuki ${ }^{53}$, N. Kazeev ${ }^{80}$, M. Kecke ${ }^{16}$, F. Keizer ${ }^{54,47}$, M. Kelsey ${ }^{67}$, M. Kenzie ${ }^{55}$, T. Ketel ${ }^{32}$, B. Khanji ${ }^{47}$, A. Kharisova ${ }^{81}$, S. Kholodenko ${ }^{43}$, K.E. Kim ${ }^{67}$, T. Kirn ${ }^{13}$, V.S. Kirsebom ${ }^{48}$, O. Kitouni ${ }^{63}$, S. Klaver ${ }^{31}$, K. Klimaszewski ${ }^{35}$, S. Koliiev ${ }^{51}$, A. Kondybayeva ${ }^{79}$, A. Konoplyannikov ${ }^{38}$, P. Kopciewicz ${ }^{34}$, R. Kopecna ${ }^{16}$, P. Koppenburg ${ }^{31}$, M. Korolev ${ }^{39}$, I. Kostiuk ${ }^{31,51}$, O. $\operatorname{Kot}^{51}$, S. Kotriakhova ${ }^{37}$, P. Kravchenko ${ }^{37}$, L. Kravchuk ${ }^{40}$, R.D. Krawczyk ${ }^{47}$, M. Kreps ${ }^{55}$, F. Kress ${ }^{60}$, S. Kretzschmar ${ }^{13}$, P. Krokovny ${ }^{42, w}$, W. Krupa ${ }^{34}$, W. Krzemien ${ }^{35}$, W. Kucewicz ${ }^{84,33, k}$, M. Kucharczyk ${ }^{33}$, V. Kudryavtsev ${ }^{42, w}$, H.S. Kuindersma ${ }^{31}$, G.J. Kunde ${ }^{66}$, T. Kvaratskheliya ${ }^{38}$, D. Lacarrere ${ }^{47}$, G. Lafferty ${ }^{61}$, A. Lai ${ }^{26}$, A. Lampis ${ }^{26}$, D. Lancierini ${ }^{49}$, J.J. Lane ${ }^{61}$, R. Lane ${ }^{53}$, G. Lanfranchi ${ }^{22}$, C. Langenbruch ${ }^{13}$, J. Langer ${ }^{14}$, O. Lantwin ${ }^{49,79}$, T. Latham ${ }^{55}$, F. Lazzari ${ }^{28, u}$, R. Le Gac ${ }^{10}$, S.H. Lee ${ }^{82}$, R. Lefèvre ${ }^{9}$, A. Leflat ${ }^{39,47}$, S. Legotin ${ }^{79}$,

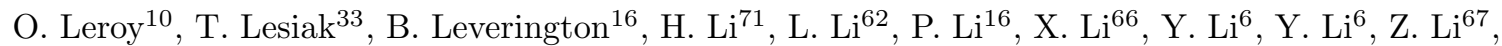

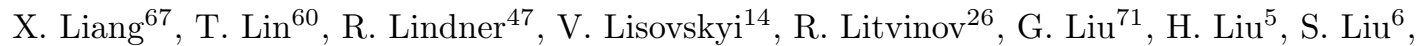
X. $\mathrm{Liu}^{3}$, A. Loi ${ }^{26}$, J. Lomba Castro ${ }^{45}$, I. Longstaff ${ }^{58}$, J.H. Lopes ${ }^{2}$, G. Loustau ${ }^{49}$, G.H. Lovell ${ }^{54}$, Y. Lu $^{6}$, D. Lucchesi ${ }^{27, n}$, S. Luchuk ${ }^{40}$, M. Lucio Martinez ${ }^{31}$, V. Lukashenko ${ }^{31}$, Y. Luo ${ }^{3}$, A. Lupato ${ }^{61}$, E. Luppi ${ }^{20, g}$, O. Lupton ${ }^{55}$, A. Lusiani ${ }^{28, s}$, X. Lyu ${ }^{5}$, L. Ma ${ }^{6}$, S. Maccolini ${ }^{19, e}$, F. Machefert ${ }^{11}$, F. Maciuc ${ }^{36}$, V. Macko ${ }^{48}$, P. Mackowiak ${ }^{14}$, S. Maddrell-Mander ${ }^{53}$, L.R. Madhan Mohan ${ }^{53}$, O. Maev $^{37}$, A. Maevskiy ${ }^{80}$, D. Maisuzenko ${ }^{37}$, M.W. Majewski ${ }^{34}$,

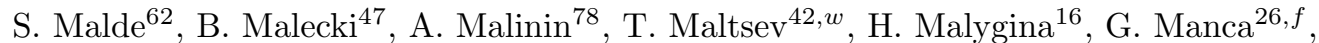
G. Mancinelli ${ }^{10}$, R. Manera Escalero ${ }^{44}$, D. Manuzzi ${ }^{19, e}$, D. Marangotto ${ }^{25, p}$, J. Maratas $^{9, v}$, J.F. Marchand ${ }^{8}$, U. Marconi ${ }^{19}$, S. Mariani ${ }^{21,47,21}$, C. Marin Benito ${ }^{11}$, M. Marinangeli ${ }^{48}$, P. Marino ${ }^{48}$, J. Marks ${ }^{16}$, P.J. Marshall ${ }^{59}$, G. Martellotti ${ }^{30}$, L. Martinazzoli ${ }^{47}$, M. Martinelli ${ }^{24, i}$, D. Martinez Santos ${ }^{45}$, F. Martinez Vidal ${ }^{46}$, A. Massafferri ${ }^{1}$, M. Materok ${ }^{13}$, R. Matev ${ }^{47}$, A. Mathad ${ }^{49}$, Z. Mathe ${ }^{47}$, V. Matiunin ${ }^{38}$, C. Matteuzzi ${ }^{24}$, K.R. Mattioli ${ }^{82}$, A. Mauri ${ }^{31}$, E. Maurice ${ }^{83,11, b}$, J. Mauricio ${ }^{44}$, M. Mazurek ${ }^{35}$, M. McCann ${ }^{60}$, L. Mcconnell ${ }^{17}$, T.H. Mcgrath ${ }^{61}$,

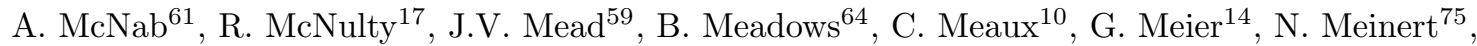
D. Melnychuk ${ }^{35}$, S. Meloni ${ }^{24, i}$, M. Merk ${ }^{31,77}$, A. Merli $^{25}$, L. Meyer Garcia ${ }^{2}$, M. Mikhasenko ${ }^{47}$, 
D.A. Milanes ${ }^{73}$, E. Millard ${ }^{55}$, M.-N. Minard ${ }^{8}$, L. Minzoni ${ }^{20, g}$, S.E. Mitchell ${ }^{57}$, B. Mitreska ${ }^{61}$, D.S. Mitzel ${ }^{47}$, A. Mödden ${ }^{14}$, R.A. Mohammed $^{62}$, R.D. Moise ${ }^{60}$, T. Mombächer ${ }^{14}$, I.A. Monroy ${ }^{73}$, S. Monteil ${ }^{9}$, M. Morandin ${ }^{27}$, G. Morello ${ }^{22}$, M.J. Morello ${ }^{28, s}$, J. Moron ${ }^{34}$, A.B. Morris ${ }^{74}$, A.G. Morris ${ }^{55}$, R. Mountain ${ }^{67}$, H. $\mathrm{Mu}^{3}$, F. Muheim ${ }^{57}$, M. Mukherjee ${ }^{7}$, M. Mulder ${ }^{47}$, D. Müller ${ }^{47}$, K. Müller ${ }^{49}$, C.H. Murphy ${ }^{62}$, D. Murray ${ }^{61}$, P. Muzzetto ${ }^{26}$, P. Naik ${ }^{53}$, T. Nakada ${ }^{48}$, R. Nandakumar ${ }^{56}$, T. Nanut ${ }^{48}$, I. Nasteva ${ }^{2}$, M. Needham ${ }^{57}$, I. Neri ${ }^{20, g}$, N. Neri ${ }^{25, p}$, S. Neubert ${ }^{74}$, N. Neufeld ${ }^{47}$, R. Newcombe ${ }^{60}$, T.D. gguyen $^{48}$, C. Nguyen-Mau ${ }^{48, m}$, E.M. Niel ${ }^{11}$, S. Nieswand ${ }^{13}$, N. Nikitin ${ }^{39}$, N.S. Nolte ${ }^{47}$, C. Nunez ${ }^{82}$, A. Oblakowska-Mucha ${ }^{34}$, V. Obraztsov ${ }^{43}$, S. Ogilvy ${ }^{58}$, D.P. O'Hanlon ${ }^{53}$, R. Oldeman ${ }^{26, f}$, C.J.G. Onderwater ${ }^{76}$, J. D. Osborn ${ }^{82}$, A. Ossowska ${ }^{33}$, J.M. Otalora Goicochea ${ }^{2}$, T. Ovsiannikova ${ }^{38}$, P. Owen ${ }^{49}$, A. Oyanguren ${ }^{46}$, B. Pagare ${ }^{55}$, P.R. Pais ${ }^{47}$, T. Pajero ${ }^{28,47, s}$, A. Palano ${ }^{18}$, M. Palutan ${ }^{22}$, Y. Pan ${ }^{61}$, G. Panshin ${ }^{81}$, A. Papanestis ${ }^{56}$, M. Pappagallo ${ }^{57}$, L.L. Pappalardo ${ }^{20, g}$, C. Pappenheimer ${ }^{64}$, W. Parker ${ }^{65}$, C. Parkes ${ }^{61}$, C.J. Parkinson ${ }^{45}$, B. Passalacqua ${ }^{20}$, G. Passaleva ${ }^{21,47}$, A. Pastore ${ }^{18}$, M. Patel ${ }^{60}$, C. Patrignani $^{19, e}$, A. Pearce ${ }^{47}$, A. Pellegrino ${ }^{31}$, M. Pepe Altarelli ${ }^{47}$, S. Perazzini ${ }^{19}$, D. Pereima ${ }^{38}$, P. Perret $^{9}$, K. Petridis ${ }^{53}$, A. Petrolini ${ }^{23, h}$, A. Petrov ${ }^{78}$, S. Petrucci ${ }^{57}$, M. Petruzzo ${ }^{25}$, A. Philippov ${ }^{41}$, L. Pica ${ }^{28}$, B. Pietrzyk ${ }^{8}$, G. Pietrzyk ${ }^{48}$, M. Pili ${ }^{62}$, D. Pinci ${ }^{30}$, J. Pinzino ${ }^{47}$, F. Pisani ${ }^{47}$, A. Piucci $^{16}$, Resmi P.K ${ }^{10}$, V. Placinta ${ }^{36}$, S. Playfer ${ }^{57}$, J. Plews ${ }^{52}$, M. Plo Casasus ${ }^{45}$, F. Polci ${ }^{12}$, M. Poli Lener ${ }^{22}$, M. Poliakova ${ }^{67}$, A. Poluektov ${ }^{10}$, N. Polukhina ${ }^{79, c}$, I. Polyakov ${ }^{67}$, E. Polycarpo ${ }^{2}$, G.J. Pomery ${ }^{53}$, S. Ponce ${ }^{47}$, A. Popov ${ }^{43}$, D. Popov ${ }^{5,47}$, S. Popov ${ }^{41}$, S. Poslavskii ${ }^{43}$, K. Prasanth ${ }^{33}$, L. Promberger ${ }^{47}$, C. Prouve ${ }^{45}$, V. Pugatch ${ }^{51}$, A. Puig Navarro ${ }^{49}$, H. Pullen ${ }^{62}$, G. Punzi ${ }^{28, o}$, W. Qian ${ }^{5}$, J. Qin ${ }^{5}$, R. Quagliani ${ }^{12}$, B. Quintana ${ }^{8}$, N.V. Raab ${ }^{17}$, R.I. Rabadan Trejo ${ }^{10}$, B. Rachwal ${ }^{34}$, J.H. Rademacker ${ }^{53}$, M. Rama ${ }^{28}$, M. Ramos Pernas ${ }^{45}$, M.S. Rangel ${ }^{2}$, F. Ratnikov ${ }^{41,80}$, G. Raven ${ }^{32}$, M. Reboud ${ }^{8}$, F. Redi ${ }^{48}$, F. Reiss ${ }^{12}$, C. Remon Alepuz ${ }^{46}$, Z. Ren $^{3}$, V. Renaudin ${ }^{62}$, R. Ribatti ${ }^{28}$, S. Ricciardi ${ }^{56}$, D.S. Richards ${ }^{56}$, K. Rinnert ${ }^{59}$, P. Robbe ${ }^{11}$, A. Robert ${ }^{12}$, G. Robertson ${ }^{57}$, A.B. Rodrigues ${ }^{48}$, E. Rodrigues ${ }^{59}$, J.A. Rodriguez Lopez ${ }^{73}$, M. Roehrken ${ }^{47}$, A. Rollings ${ }^{62}$, P. Roloff ${ }^{47}$, V. Romanovskiy ${ }^{43}$, M. Romero Lamas ${ }^{45}$, A. Romero Vidal ${ }^{45}$, J.D. Roth ${ }^{82}$, M. Rotondo ${ }^{22}$, M.S. Rudolph ${ }^{67}$, T. Ruf ${ }^{47}$, J. Ruiz Vidal ${ }^{46}$, A. Ryzhikov ${ }^{80}$, J. Ryzka ${ }^{34}$, J.J. Saborido Silva ${ }^{45}$, N. Sagidova ${ }^{37}$, N. Sahoo ${ }^{55}$, B. Saitta ${ }^{26, f}$, C. Sanchez Gras ${ }^{31}$, C. Sanchez Mayordomo ${ }^{46}$, R. Santacesaria ${ }^{30}$, C. Santamarina Rios ${ }^{45}$, M. Santimaria ${ }^{22}$, E. Santovetti ${ }^{29, j}$, D. Saranin ${ }^{79}$, G. Sarpis ${ }^{61}$, M. Sarpis ${ }^{74}$, A. Sarti ${ }^{30}$, C. Satriano ${ }^{30, r}$, A. Satta ${ }^{29}$, M. Saur ${ }^{5}$, D. Savrina ${ }^{38,39}$, H. Sazak ${ }^{9}$, L.G. Scantlebury Smead ${ }^{62}$, S. Schael ${ }^{13}$, M. Schellenberg ${ }^{14}$, M. Schiller ${ }^{58}$, H. Schindler ${ }^{47}$, M. Schmelling ${ }^{15}$, T. Schmelzer ${ }^{14}$, B. Schmidt ${ }^{47}$, O. Schneider ${ }^{48}$, A. Schopper ${ }^{47}$, M. Schubiger ${ }^{31}$, S. Schulte ${ }^{48}$, M.H. Schune ${ }^{11}$, R. Schwemmer ${ }^{47}$, B. Sciascia ${ }^{22}$, A. Sciubba ${ }^{22}$, S. Sellam ${ }^{68}$, A. Semennikov ${ }^{38}$, A. Sergi ${ }^{52,47}$, N. Serra ${ }^{49}$, J. Serrano ${ }^{10}$, L. Sestini ${ }^{27}$, A. Seuthe ${ }^{14}$, P. Seyfert ${ }^{47}$, D.M. Shangase ${ }^{82}$, M. Shapkin ${ }^{43}$, I. Shchemerov ${ }^{79}$, L. Shchutska ${ }^{48}$, T. Shears ${ }^{59}$, L. Shekhtman ${ }^{42, w}$, V. Shevchenko ${ }^{78}$, E.B. Shields ${ }^{24, i}$, E. Shmanin ${ }^{79}$, J.D. Shupperd ${ }^{67}$, B.G. Siddi ${ }^{20}$, R. Silva Coutinho ${ }^{49}$ L. Silva de Oliveira ${ }^{2}$, G. Simi ${ }^{27}$, S. Simone ${ }^{18, d}$, I. Skiba ${ }^{20, g}$, N. Skidmore ${ }^{74}$, T. Skwarnicki ${ }^{67}$,

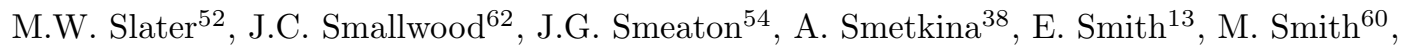
A. Snoch ${ }^{31}$, M. Soares ${ }^{19}$, L. Soares Lavra ${ }^{9}$, M.D. Sokoloff ${ }^{64}$, F.J.P. Soler ${ }^{58}$, A. Solovev ${ }^{37}$, I. Solovyev ${ }^{37}$, F.L. Souza De Almeida ${ }^{2}$, B. Souza De Paula ${ }^{2}$, B. Spaan ${ }^{14}$, E. Spadaro Norella ${ }^{25, p}$, P. Spradlin ${ }^{58}$, F. Stagni ${ }^{47}$, M. Stahl ${ }^{64}$, S. Stahl ${ }^{47}$, P. Stefko ${ }^{48}$, O. Steinkamp ${ }^{49,79}$, S. Stemmle ${ }^{16}$, O. Stenyakin ${ }^{43}$, H. Stevens ${ }^{14}$, S. Stone ${ }^{67}$, S. Stracka ${ }^{28}$, M.E. Stramaglia ${ }^{48}$, M. Straticiuc ${ }^{36}$, D. Strekalina ${ }^{79}$, S. Strokov ${ }^{81}$, F. Suljik ${ }^{62}$, J. Sun ${ }^{26}$, L. Sun ${ }^{72}$, Y. Sun ${ }^{65}$, P. Svihra ${ }^{61}$, P.N. Swallow ${ }^{52}$, K. Swientek ${ }^{34}$, A. Szabelski ${ }^{35}$, T. Szumlak ${ }^{34}$, M. Szymanski ${ }^{47}$, S. Taneja ${ }^{61}$, Z. Tang ${ }^{3}$, T. Tekampe ${ }^{14}$, F. Teubert ${ }^{47}$, E. Thomas $^{47}$, K.A. Thomson ${ }^{59}$, M.J. Tilley ${ }^{60}$, V. Tisserand ${ }^{9}$, S. T'Jampens ${ }^{8}$, M. Tobin ${ }^{6}$, S. Tolk ${ }^{47}$, L. Tomassetti ${ }^{20, g}$, D. Torres Machado ${ }^{1}$,

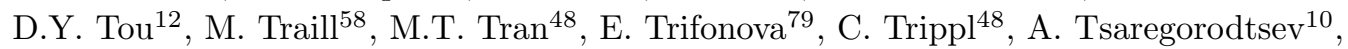




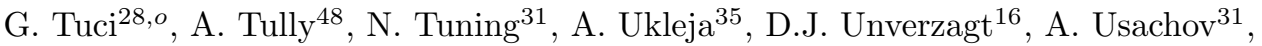

A. Ustyuzhanin ${ }^{41,80}$, U. Uwer ${ }^{16}$, A. Vagner ${ }^{81}$, V. Vagnoni ${ }^{19}$, A. Valassi ${ }^{47}$, G. Valenti ${ }^{19}$,

M. van Beuzekom ${ }^{31}$, H. Van Hecke ${ }^{66}$, E. van Herwijnen ${ }^{79}$, C.B. Van Hulse ${ }^{17}$, M. van Veghel ${ }^{76}$,

R. Vazquez Gomez ${ }^{45}$, P. Vazquez Regueiro ${ }^{45}$, C. Vázquez Sierra ${ }^{31}$, S. Vecchi ${ }^{20}$, J.J. Velthuis ${ }^{53}$,

M. Veltri ${ }^{21, q}$, A. Venkateswaran ${ }^{67}$, M. Veronesi ${ }^{31}$, M. Vesterinen ${ }^{55}$, D. Vieira ${ }^{64}$, M. Vieites Diaz ${ }^{48}$,

H. Viemann ${ }^{75}$, X. Vilasis-Cardona ${ }^{44}$, E. Vilella Figueras ${ }^{59}$, P. Vincent ${ }^{12}$, G. Vitali ${ }^{28}$,

A. Vitkovskiy ${ }^{31}$, A. Vollhardt ${ }^{49}$, D. Vom Bruch ${ }^{12}$, A. Vorobyev ${ }^{37}$, V. Vorobyev ${ }^{42, w}$, N. Voropaev ${ }^{37}$,

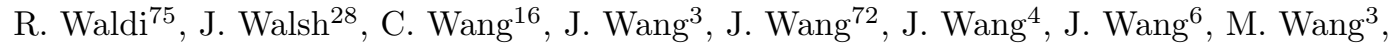

R. Wang ${ }^{53}$, Y. Wang ${ }^{7}$, Z. Wang ${ }^{49}$, D.R. Ward ${ }^{54}$, H.M. Wark ${ }^{59}$, N.K. Watson ${ }^{52}$, S.G. Weber ${ }^{12}$,

D. Websdale ${ }^{60}$, C. Weisser ${ }^{63}$, B.D.C. Westhenry ${ }^{53}$, D.J. White ${ }^{61}$, M. Whitehead ${ }^{53}$, D. Wiedner ${ }^{14}$,

G. Wilkinson ${ }^{62}$, M. Wilkinson ${ }^{67}$, I. Williams ${ }^{54}$, M. Williams ${ }^{63,69}$, M.R.J. Williams ${ }^{61}$,

F.F. Wilson ${ }^{56}$, W. Wislicki ${ }^{35}$, M. Witek ${ }^{33}$, L. Witola ${ }^{16}$, G. Wormser ${ }^{11}$, S.A. Wotton ${ }^{54}, \mathrm{H} \mathrm{Wu}^{67}$,

K. Wyllie ${ }^{47}$, Z. Xiang ${ }^{5}$, D. Xiao ${ }^{7}$, Y. Xie ${ }^{7}$, H. Xing ${ }^{71}$, A. Xu ${ }^{4}$, J. Xu ${ }^{5}$, L. Xu ${ }^{3}$, M. Xu ${ }^{7}$, Q. Xu ${ }^{5}$, Z. Xu ${ }^{4}$, D. Yang ${ }^{3}$, Y. Yang ${ }^{5}$, Z. Yang ${ }^{3}$, Z. Yang ${ }^{65}$, Y. Yao ${ }^{67}$, L.E. Yeomans ${ }^{59}$, H. Yin ${ }^{7}$, J. Yu ${ }^{7}$, X. Yuan ${ }^{67}$, O. Yushchenko ${ }^{43}$, K.A. Zarebski ${ }^{52}$, M. Zavertyaev ${ }^{15, c}$, M. Zdybal ${ }^{33}$, O. Zenaiev ${ }^{47}$, M. Zeng ${ }^{3}$, D. Zhang ${ }^{7}$, L. Zhang ${ }^{3}$, S. Zhang ${ }^{4}$, Y. Zhang ${ }^{47}$, A. Zhelezov ${ }^{16}$, Y. Zheng ${ }^{5}$, X. Zhou ${ }^{5}$, Y. Zhou ${ }^{5}$, X. Zhu ${ }^{3}$, V. Zhukov ${ }^{13,39}$, J.B. Zonneveld ${ }^{57}$, S. Zucchelli ${ }^{19, e}$, D. Zuliani ${ }^{27}$, G. Zunica ${ }^{61}$

1 Centro Brasileiro de Pesquisas Físicas (CBPF), Rio de Janeiro, Brazil

2 Universidade Federal do Rio de Janeiro (UFRJ), Rio de Janeiro, Brazil

3 Center for High Energy Physics, Tsinghua University, Beijing, China

4 School of Physics State Key Laboratory of Nuclear Physics and Technology, Peking University, Beijing, China

5 University of Chinese Academy of Sciences, Beijing, China

6 Institute Of High Energy Physics (IHEP), Beijing, China

7 Institute of Particle Physics, Central China Normal University, Wuhan, Hubei, China

8 Univ. Grenoble Alpes, Univ. Savoie Mont Blanc, CNRS, IN2P3-LAPP, Annecy, France

9 Université Clermont Auvergne, CNRS/IN2P3, LPC, Clermont-Ferrand, France

10 Aix Marseille Univ, CNRS/IN2P3, CPPM, Marseille, France

11 Université Paris-Saclay, CNRS/IN2P3, IJCLab, Orsay, France

12 LPNHE, Sorbonne Université, Paris Diderot Sorbonne Paris Cité, CNRS/IN2P3, Paris, France

13 I. Physikalisches Institut, RWTH Aachen University, Aachen, Germany

14 Fakultät Physik, Technische Universität Dortmund, Dortmund, Germany

15 Max-Planck-Institut für Kernphysik (MPIK), Heidelberg, Germany

16 Physikalisches Institut, Ruprecht-Karls-Universität Heidelberg, Heidelberg, Germany

17 School of Physics, University College Dublin, Dublin, Ireland

18 INFN Sezione di Bari, Bari, Italy

19 INFN Sezione di Bologna, Bologna, Italy

20 INFN Sezione di Ferrara, Ferrara, Italy

21 INFN Sezione di Firenze, Firenze, Italy

22 INFN Laboratori Nazionali di Frascati, Frascati, Italy

23 INFN Sezione di Genova, Genova, Italy

24 INFN Sezione di Milano-Bicocca, Milano, Italy

25 INFN Sezione di Milano, Milano, Italy

26 INFN Sezione di Cagliari, Monserrato, Italy

27 Universita degli Studi di Padova, Universita e INFN, Padova, Padova, Italy

28 INFN Sezione di Pisa, Pisa, Italy

29 INFN Sezione di Roma Tor Vergata, Roma, Italy

30 INFN Sezione di Roma La Sapienza, Roma, Italy

31 Nikhef National Institute for Subatomic Physics, Amsterdam, Netherlands 
Nikhef National Institute for Subatomic Physics and VU University Amsterdam, Amsterdam, Netherlands

33 Henryk Niewodniczanski Institute of Nuclear Physics Polish Academy of Sciences, Kraków, Poland

${ }^{34}$ AGH - University of Science and Technology, Faculty of Physics and Applied Computer Science, Kraków, Poland

35 National Center for Nuclear Research (NCBJ), Warsaw, Poland

36 Horia Hulubei National Institute of Physics and Nuclear Engineering, Bucharest-Magurele, Romania

37 Petersburg Nuclear Physics Institute NRC Kurchatov Institute (PNPI NRC KI), Gatchina, Russia

38 Institute of Theoretical and Experimental Physics NRC Kurchatov Institute (ITEP NRC KI), Moscow, Russia, Moscow, Russia

39 Institute of Nuclear Physics, Moscow State University (SINP MSU), Moscow, Russia

40 Institute for Nuclear Research of the Russian Academy of Sciences (INR RAS), Moscow, Russia

41 Yandex School of Data Analysis, Moscow, Russia

${ }^{42}$ Budker Institute of Nuclear Physics (SB RAS), Novosibirsk, Russia

43 Institute for High Energy Physics NRC Kurchatov Institute (IHEP NRC KI), Protvino, Russia, Protvino, Russia

${ }^{44}$ ICCUB, Universitat de Barcelona, Barcelona, Spain

45 Instituto Galego de Física de Altas Enerxías (IGFAE), Universidade de Santiago de Compostela, Santiago de Compostela, Spain

46 Instituto de Fisica Corpuscular, Centro Mixto Universidad de Valencia - CSIC, Valencia, Spain

47 European Organization for Nuclear Research (CERN), Geneva, Switzerland

48 Institute of Physics, Ecole Polytechnique Fédérale de Lausanne (EPFL), Lausanne, Switzerland

49 Physik-Institut, Universität Zürich, Zürich, Switzerland

50 NSC Kharkiv Institute of Physics and Technology (NSC KIPT), Kharkiv, Ukraine

51 Institute for Nuclear Research of the National Academy of Sciences (KINR), Kyiv, Ukraine

52 University of Birmingham, Birmingham, United Kingdom

53 H.H. Wills Physics Laboratory, University of Bristol, Bristol, United Kingdom

${ }^{54}$ Cavendish Laboratory, University of Cambridge, Cambridge, United Kingdom

55 Department of Physics, University of Warwick, Coventry, United Kingdom

56 STFC Rutherford Appleton Laboratory, Didcot, United Kingdom

57 School of Physics and Astronomy, University of Edinburgh, Edinburgh, United Kingdom

58 School of Physics and Astronomy, University of Glasgow, Glasgow, United Kingdom

59 Oliver Lodge Laboratory, University of Liverpool, Liverpool, United Kingdom

60 Imperial College London, London, United Kingdom

61 Department of Physics and Astronomy, University of Manchester, Manchester, United Kingdom

${ }^{62}$ Department of Physics, University of Oxford, Oxford, United Kingdom

${ }^{63}$ Massachusetts Institute of Technology, Cambridge, MA, United States

${ }^{64}$ University of Cincinnati, Cincinnati, OH, United States

65 University of Maryland, College Park, MD, United States

66 Los Alamos National Laboratory (LANL), Los Alamos, United States

67 Syracuse University, Syracuse, NY, United States

68 Laboratory of Mathematical and Subatomic Physics, Constantine, Algeria, associated to ${ }^{2}$

${ }^{69}$ School of Physics and Astronomy, Monash University, Melbourne, Australia, associated to ${ }^{55}$

70 Pontifícia Universidade Católica do Rio de Janeiro (PUC-Rio), Rio de Janeiro, Brazil, associated to ${ }^{2}$

71 Guangdong Provencial Key Laboratory of Nuclear Science, Institute of Quantum Matter, South China Normal University, Guangzhou, China, associated to ${ }^{3}$

72 School of Physics and Technology, Wuhan University, Wuhan, China, associated to ${ }^{3}$

73 Departamento de Fisica, Universidad Nacional de Colombia, Bogota, Colombia, associated to ${ }^{12}$

74 Universität Bonn - Helmholtz-Institut für Strahlen und Kernphysik, Bonn, Germany, associated to ${ }^{16}$ 
75 Institut für Physik, Universität Rostock, Rostock, Germany, associated to ${ }^{16}$

76 Van Swinderen Institute, University of Groningen, Groningen, Netherlands, associated to ${ }^{31}$

77 Universiteit Maastricht, Maastricht, Netherlands, associated to ${ }^{31}$

78 National Research Centre Kurchatov Institute, Moscow, Russia, associated to ${ }^{38}$

79 National University of Science and Technology "MISIS", Moscow, Russia, associated to ${ }^{38}$

80 National Research University Higher School of Economics, Moscow, Russia, associated to ${ }^{41}$

81 National Research Tomsk Polytechnic University, Tomsk, Russia, associated to ${ }^{38}$

82 University of Michigan, Ann Arbor, United States, associated to ${ }^{67}$

83 Laboratoire Leprince-Ringuet, Palaiseau, France

${ }^{84}$ AGH - University of Science and Technology, Faculty of Computer Science, Electronics and Telecommunications, Kraków, Poland

a Universidade Federal do Triângulo Mineiro (UFTM), Uberaba-MG, Brazil

${ }^{b}$ Laboratoire Leprince-Ringuet, Palaiseau, France

c P.N. Lebedev Physical Institute, Russian Academy of Science (LPI RAS), Moscow, Russia

d Università di Bari, Bari, Italy

e Università di Bologna, Bologna, Italy

$f$ Università di Cagliari, Cagliari, Italy

$g$ Università di Ferrara, Ferrara, Italy

$h$ Università di Genova, Genova, Italy

i Università di Milano Bicocca, Milano, Italy

j Università di Roma Tor Vergata, Roma, Italy

${ }^{k}$ AGH - University of Science and Technology, Faculty of Computer Science, Electronics and Telecommunications, Kraków, Poland

${ }^{l}$ DS4DS, La Salle, Universitat Ramon Llull, Barcelona, Spain

$m$ Hanoi University of Science, Hanoi, Vietnam

$n$ Università di Padova, Padova, Italy

- Università di Pisa, Pisa, Italy

p Università degli Studi di Milano, Milano, Italy

$q$ Università di Urbino, Urbino, Italy

$r$ Università della Basilicata, Potenza, Italy

$s$ Scuola Normale Superiore, Pisa, Italy

${ }^{t}$ Università di Modena e Reggio Emilia, Modena, Italy

u Università di Siena, Siena, Italy

$v$ MSU - Iligan Institute of Technology (MSU-IIT), Iligan, Philippines

w Novosibirsk State University, Novosibirsk, Russia

$x$ INFN Sezione di Trieste, Trieste, Italy

y Universidad Nacional Autonoma de Honduras, Tegucigalpa, Honduras 